\title{
Impedance and Instability Threshold Estimates in the Main Injector I
}

\author{
M.A. Martens and K.Y. Ng \\ Fermi National Accelerator Laboratory \\ P.O. Box 500, Batavia, Illinois 60510
}

March 1994 


\section{Disclaimer}

This report was prepared as an account of work sponsored by an agency of the United States Government. Neither the United States Government nor any agency thereof, nor any of their employees, makes any warranty, express or implied, or assumes any legal liability or responsibility for the accuracy, completeness, or usefulness of any information, apparatus, product, or process disclosed, or represents that its use would not infringe privately owned rights. Reference herein to any specific commercial product, process, or service by trade name, trademark, manufacturer, or otherwise, does not necessarily constitute or imply its endorsement, recommendation, or favoring by the United States Government or any agency thereof. The views and opinions of authors expressed herein do not necessarily state or reflect those of the United States Government or any agency there of. 


\title{
Impedance and Instability Threshold Estimates in the Main Injector I
}

\author{
M.A. Martens and K.Y. Ng
}

Fermi National Accelerator Laboratory

P.O. Box 500, Batavia, Illinois 60510 


\section{Contents}

I Introduction $\quad \mathbf{3}$

II Impedance and Stability Estimations 4

A Space Charge Impedance . . . . . . . . . . . . . . . . 4

B Space Charge Tune Shifts .............. 7

C RF Cavities and Coupled Bunch Instabilities . . . . . . . 12

D Resistive Wall . . . . . . . . . . . . 20

E Beam Position Monitors .............. 25

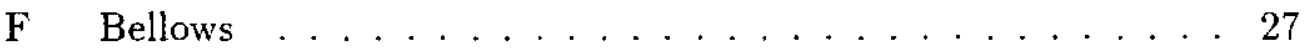

G Vacuum Valves ................... 29

H Lambertsons .................... . . . . . . . . .

I Microwave Instabilities . . . . . . . . . . . . 35

III Conclusions and Further Work 42 


\section{List of Figures}

1 Geometry of Main Injector Beampipe and Beam Position Monitor. ..................... 5

2 Longitudinal form factor for elliptical phase space density distribution. ..................... 17

3 Transverse coupled bunch instability form factor as function of $\alpha$. (See Equation 19) . . . . . . . . . . 20

4 Real part of the longitudinal impedance for the circular bellows with 10 convolutions. . . . . . . . . . . 28

5 Real part of the transverse impedance for the circular bellows with 10 convolutions. . . . . . . . . . . . . 28

6 Approximate geometry of Main Injector vacuum valve with pillbox cavities. .................. 30

7 Approximate geometry of Main Injector vacuum valve without pillbox cavities. .................. . . 33

8 Real and imaginary part of the longitudinal impedance for the Lambertson magnets. . . . . . . . . . . . . 36

9 Real and imaginary part of the transverse impedance for the Lambertson magnets. . . . . . . . . . . . . . 36 


\section{Introduction}

One of the important considerations in the design of the Main Injector is the beam coupling impedances in the vacuum chamber and the stability of the beam. Along with the higher intensities comes the possibility of instabilities which lead to growth in beam emittances and/or the loss of beam. This paper makes estimations of the various impedances and instability thresholds based on impedance estimations and measurements. Notably missing from this paper is any analysis of transition crossing and its potential limitations on beam intensity and beam emittance. Future work should consider this issue.

The body of the work contains detailed analysis of the various impedance estimations and instability threshold calculations. The calculations are based on the Main Injector beam intensity of $6 \times 10^{10}$ protons per bunch, $95 \%$ normalized transverse emittances of $20 \pi \mathrm{mm}-\mathrm{mrad}$, and $95 \%$ normalized longitudinal emittance of $0.1 \mathrm{eV}$-s at $8.9 \mathrm{GeV}$ injection energy and $0.25 \mathrm{eV}$-s at $150 \mathrm{GeV}$ flattop energy.

The conclusions section summarizes the results in the paper and is meant to be readable by itself without referring to the rest of the paper. Also in the conclusion section are recommendations for future investigations. 


\section{Impedance and Stability Estimations}

\section{A Space Charge Impedance}

The Main Injector beampipe, shown in Figure 1, is approximately elliptical with a full height of $5.31 \mathrm{~cm}$ (2.09 inches) and a full width of $12.3 \mathrm{~cm}$ (4.84 inches). When installed in the Main Injector magnets and under vacuum the beampipe deforms reducing its height to $5.08 \mathrm{~cm}$. The elliptical beampipe shape makes calculation of the space charge impedance difficult so we make estimations using beampipes with circular and rectangular cross sections instead. The formula for circular cross sections is well known while the formula for rectangular cross sections is derived using conformal mapping techniques [1].

For a circular beampipe of radius $b$ and a cylindrical beam of radius $a$ located at the center of the beampipe, the longitudinal impedance per harmonic is

$$
\frac{Z_{\|}(\omega)}{n}=-j \frac{Z_{o}}{2 \beta \gamma^{2}}[1+2 \ln (b / a)]
$$

and the transverse impedance is

$$
Z_{\perp}(\omega)=-j \frac{R Z_{o}}{\beta^{2} \gamma^{2}}\left(\frac{1}{a^{2}}-\frac{1}{b^{2}}\right)
$$

where $n$ is the harmonic number, $R=528.3 \mathrm{~m}$ is the radius of the machine, and $Z_{o}=377 \Omega$ is the free-space impedance.

For a rectangular beampipe with full height $h$ and full width $w$ the longitudinal impedance per harmonic [1] is

$$
\frac{Z_{\| l}(\omega)}{n}=-j \frac{Z_{o}}{2 \beta \gamma^{2}}\left[1+2 \ln \left(\frac{2 h}{\pi a} \tanh \left(\frac{\pi w}{2 h}\right)\right)\right]
$$




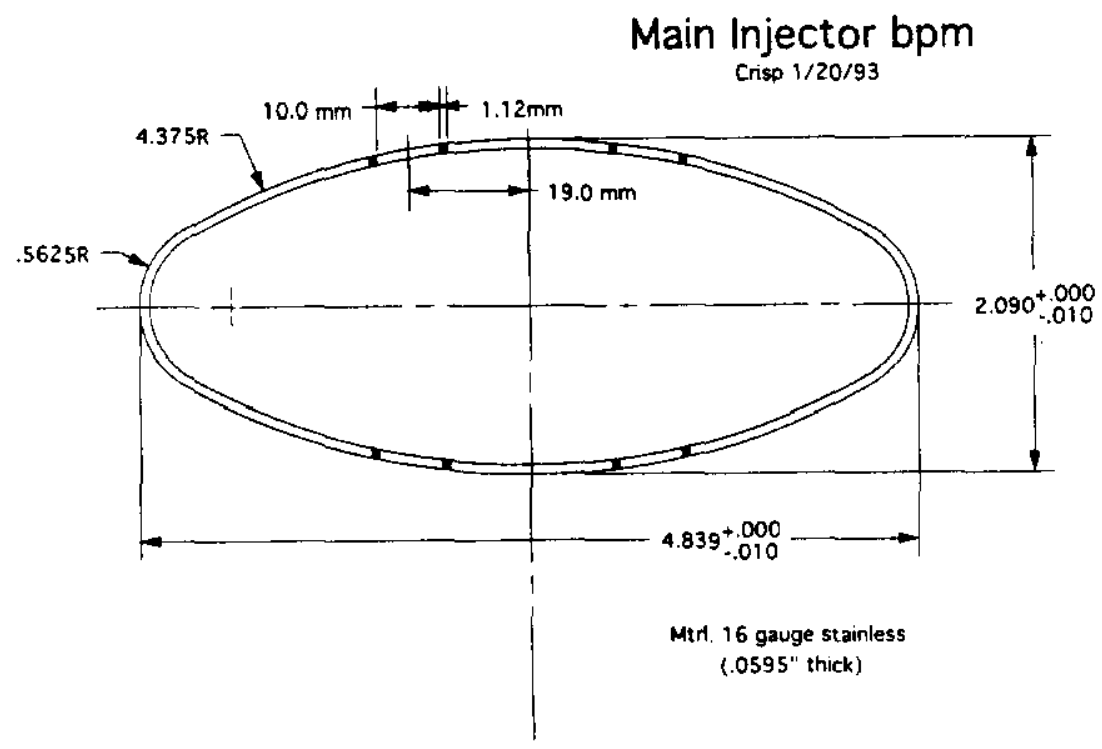

Figure 1: Geometry of Main Injector Beampipe and Beam Position Monitor.

and the transverse impedance is

$$
Z_{H, V}(\omega)=-j \frac{R Z_{0}}{\beta^{2} \gamma^{2}}\left[\frac{1}{a^{2}}-\frac{8}{h^{2}}\left(\xi_{1}^{H, V}-\epsilon_{1}^{H, V}\right)\right]
$$

where the superscripts $H$ and $V$ refer to the horizontal and vertical direction and $\epsilon_{1}$ and $\xi_{1}$, called the incoherent and coherent non-penetrating electric image coefficients, are found from the conformal mapping technique and depend on the ratio $w / h$. For the ratio $w / h=2.51$ we find $\xi_{1}^{\mathrm{H}}-\epsilon_{1}^{\mathrm{H}}=0.208$ and $\xi_{1}^{\mathrm{V}}-\epsilon_{1}^{\mathrm{V}}=0.411$. The space charge impedances given by the above formulas are valid up to angular frequency $\omega<\gamma c / b, b$ being the beampipe radius, and roll off at higher frequencies.

Using Equations 1-4 we calculate the space charge impedance at $8.9 \mathrm{GeV}$ for beam with a $95 \%$ normalized transverse emittance of $20 \pi$ mm-mrad. A 


\begin{tabular}{|c|c|c|}
\hline & $\begin{array}{c}\text { Circular } \\
\text { Beampipe }\end{array}$ & $\begin{array}{c}\text { Rectangular } \\
\text { Beampipe }\end{array}$ \\
\hline$Z_{\|} / n$ & $-j 11.3 \Omega$ & $-j 12.3 \Omega$ \\
\hline$Z_{\perp x}$ & $-j 314 \mathrm{M} \Omega / \mathrm{m}$ & $-j 317 \mathrm{M} \Omega / \mathrm{m}$ \\
\hline$Z_{\perp y}$ & $-j 302 \mathrm{M} \Omega / \mathrm{m}$ & $-j 303 \mathrm{M} \Omega / \mathrm{m}$ \\
\hline
\end{tabular}

Table 1: Space charge impedance for circular beampipe with $2.39 \mathrm{~cm}$ radius and rectangular beampipe $4.78 \mathrm{~cm}$ by $12.0 \mathrm{~cm}$. Calculations are at $8.9 \mathrm{Gev}$ using a cylindrically uniform beam with transverse radius of $2.65 \mathrm{~mm}$ in the $x$ direction and $2.70 \mathrm{~mm}$ in the $y$ direction.

Gaussian beam distribution in transverse phase space is assumed corresponding to a transverse beam size of $\sigma_{x}=2.65 \mathrm{~mm}$ and $\sigma_{y}=2.70 \mathrm{~mm}$ at points of average beta, $\beta_{x, \text { avg }}=20 \mathrm{~m}$ and $\beta_{y, \text { avg }}=20.1 \mathrm{~m}$. We therefore use a cylindrical beam with a radius of $a=\sigma$ in our calculation of the space charge impedances. In the circular case the beampipe has a radius of $b=2.39 \mathrm{~cm}$ while in the rectangular case the beampipe has a full height of $h=4.78 \mathrm{~cm}$ and a full width of $w=12.0 \mathrm{~cm}$. The results of these calculations are shown in Table 1.

As will be shown in Section I, the longitudinal space charge impedance per harmonic is below the threshold of microwave instability, except in the region of transition crossing where the Landau damping due to revolution frequency spread becomes negligibly small. The growth rate without Landau damping can be shown to be proportional to $Z_{\|}$. Thus the microwave 
growth due to space charge near transition will be tremendous near $\omega / 2 \pi \approx$ $\gamma_{t} c / 2 \pi b \sim 40 \mathrm{GHz}$ where the transition gamma is $\gamma_{t}=20.4$. On the other hand, however, the transverse impedance due to space charge will not cause any microwave instability. As will be shown in Section I, there is always Landau damping due to tune spread although the revolution frequency spread become negligibly small. The full issues of transition crossing are not considered in this paper.

\section{B Space Charge Tune Shifts}

We are also interested in the coherent and incoherent tune shifts resulting from direct space charge forces, electric image charges, and magnetic image currents. These image charges and image currents inside the vacuum chamber walls and magnet laminations create electromagnetic fields which alter the transverse focusing force on the particles thus changing their tune. The tune shifts are usually characterized $[2,3]$ by the electrostatic coherent and incoherent image coefficients $\xi_{1}^{\mathrm{H}, \mathrm{V}}$ and $\epsilon_{1}^{\mathrm{H}, \mathrm{V}}$ and by the magnetic coherent and incoherent image coefficients $\xi_{2}^{\mathrm{H}, \mathrm{V}}$ and $\epsilon_{2}^{\mathrm{H}, \mathrm{V}}$ which all depend on the geometry of the vacuum chamber and magnet laminations.

The total tune shift is the sum of the tune shifts from the various image fields. These include electric fields from charge induced on the vacuum chamber wall, magnetic fields from image currents induced in the magnets by $\mathrm{DC}$ beam current, and magnetic fields from image currents induced in the vacuum chamber walls by the $\mathrm{AC}$ beam current. The $\mathrm{AC}$ beam current is a result of both longitudinal bunching and transverse betatron motion. 


\begin{tabular}{|c|c|c|c|}
\hline \multicolumn{2}{|c|}{} & Horizontal & Vertical \\
\hline Electric coherent & $\xi_{1}$ & +0.0037 & +0.615 \\
\hline Electric incoherent & $\epsilon_{1}$ & -0.204 & +0.204 \\
\hline Magnetic coherent & $\xi_{2}$ & +0.0 & +0.617 \\
\hline Magnetic incoherent & $\epsilon_{2}$ & -0.411 & +0.411 \\
\hline
\end{tabular}

Table 2: Electric image coefficients for an elliptical beampipe with width to height ratio of 2.51 and magnetic image coefficients for a set of parallel magnet poles.

The magnetic fields from the $\mathrm{AC}$ beam current do not penetrate the vacuum chamber while those from the $\mathrm{DC}$ beam current do penetrate the vacuum chamber walls and enter the magnet laminations.

Including all of the above contributions to the tune shift [4] leads to the following expression for the total coherent tune shift

$$
\begin{aligned}
\Delta \nu_{\text {coh }}^{\mathrm{H}, \mathrm{V}}=-\frac{4 N r_{o} R}{\pi \nu_{\mathrm{H}, \mathrm{V}} \gamma} & \left(\frac{\xi_{1}^{\mathrm{H}, \mathrm{V}}}{\beta^{2} B h^{2}}+\frac{\epsilon_{2}^{\mathrm{H}, \mathrm{V}}}{g^{2}} \mathcal{F}\right. \\
& \left.-\frac{\xi_{1}^{\mathrm{H}, \mathrm{V}}\left(\frac{1}{B}-1\right)}{h^{2}}-\frac{\xi_{1}^{\mathrm{H}, \mathrm{V}}-\epsilon_{1}^{\mathrm{H}, \mathrm{V}}}{h^{2}}\right) .
\end{aligned}
$$

The first term is due to the electric image force from the wall, the second is due to the DC magnetic image force from the dipole pole faces, the third and fourth are due to the $\mathrm{AC}$ magnetic image forces from the wall due to the longitudinal bunching and the transverse beam motion, respectively. In Equation $5, N$ is the total number of particles, $r_{o}=1.535 \times 10^{-18} \mathrm{~m}$ is the classical proton radius, $R$ is the radius of the ring, $h$ is the full height of the 
beampipe, $g$ is the full magnet pole gap distance, $\mathcal{F}$ is fraction of the ring circumference covered by dipole magnets, and $\nu^{\mathrm{H}}$ and $\nu^{\mathrm{V}}$ are the horizontal and vertical tunes. The bunching factor $B$ is the ratio of the average current $I_{\text {avg }}=M I_{b}$ to the peak current $I_{p}$ and is related to both the number of bunches and the bunch length.

Similarly the incoherent tune shift is given by the expression [4]

$$
\begin{aligned}
\Delta \nu_{\text {inc }}^{\mathrm{H}, \mathrm{V}}= & -\frac{4 N r_{o} R}{\pi \nu_{\mathrm{H}, \mathrm{V} \gamma}}\left(\frac{\epsilon_{1}^{\mathrm{H}, \mathrm{V}}}{\beta^{2} B h^{2}}+\frac{\epsilon_{2}^{\mathrm{H}, \mathrm{V}}}{g^{2}} \mathcal{F}-\frac{\epsilon_{1}^{\mathrm{H}, \mathrm{V}}\left(\frac{1}{B}-1\right)}{h^{2}}\right) \\
& -\frac{N r_{o}}{4 \gamma^{2} \beta} \frac{6}{\epsilon_{N, 95 \%}} \frac{1}{B}
\end{aligned}
$$

where $\epsilon_{N, 95 \%}$ is the $95 \%$ normalized transverse emittance. The incoherent tune shift does not have a contribution from the $\mathrm{AC}$ magnetic field created by the transverse motion of the beam similar to the fourth term in Equation 5. However, there is an extra term, the last term of Equation 6, which represents the contribution from the direct space charge forces and is dependent on the transverse emittance of the beam [5]. Here a Gaussian distribution in both the longitudinal and vertical directions has been assumed.

We apply Equations 5 and 6 to the Main Injector with the design beam intensity and emittance. The total number of particles is $N=3 \times 10^{13}$, the ring radius is $R=528.3 \mathrm{~m}, h=4.78 \mathrm{~cm}$ is the height of the beampipe, $g=5.08 \mathrm{~cm}$ is the magnet pole gap distance, and $\mathcal{F}=0.543$ is fraction of the Main Injector circumference covered by dipole magnets. The tunes are $\nu^{\mathrm{H}}=26.4$ and $\nu^{\mathrm{V}}=25.4$ and the $95 \%$ normalized transverse emittance is $\epsilon_{N, 95 \%}=20 \pi$ mm-mrad. With a $95 \%$ normalized longitudinal emittance of $0.1 \mathrm{eV}$-s and an RF voltage of $400 \mathrm{kV}$ the bunching factor is $B=0.19$. 
The electric coherent and incoherent image coefficients for an elliptical beampipe with width to height ratio of 2.51 were found using a conformal mapping technique [6]. The values for the coefficients are listed in Table 2 and are not much different from the coefficients of parallel plates. The magnetic coherent and incoherent image coefficients are those of two parallel and infinitely wide magnet poles [6]. The tune shifts from the various contributions are listed in Table 3 . The net tune shifts are small enough that we do not expect any problems.

Since the Main Injector will have different operating cycles, it is useful to express the tune shifts in terms of the number of particles per bunch $N_{b}$, the total number of bunches in the ring $M$, and the total bunch length $\tau$. Using the fact that the bunching factor is

$$
B=\frac{M \tau \beta c}{3 \pi R}
$$

for the elliptical longitudinal phase space distribution (see Equation 14), we can rewrite Equations 5 and 6 in the form

$$
\begin{aligned}
& \Delta \nu_{\text {coh }}^{\mathrm{H}}=-1.26 \times 10^{-5} \frac{N_{b}}{\tau_{\text {ns }}}+7.24 \times 10^{-6} N_{b} M, \\
& \Delta \nu_{\text {coh }}^{\mathrm{V}}=-2.16 \times 10^{-3} \frac{N_{b}}{\tau_{\mathrm{ns}}}+7.53 \times 10^{-6} N_{b} M, \\
& \Delta \nu_{\text {inc }}^{\mathrm{H}}=\left(+6.90 \times 10^{-4}-\frac{4.30}{\epsilon_{N, 95 \%}}\right) \frac{N_{b}}{\tau_{\mathrm{ns}}}+7.24 \times 10^{-6} N_{b} M, \\
& \Delta \nu_{\text {inc }}^{\mathrm{V}}=\left(-7.17 \times 10^{-4}-\frac{4.30}{\epsilon_{N, 95 \%}}\right) \frac{N_{b}}{\tau_{\mathrm{ns}}}+7.53 \times 10^{-6} N_{b} M,
\end{aligned}
$$

where $\tau_{\mathrm{ns}}$ is the total bunch length in ns and $N_{b}$ is the number of particles per bunch in units of $10^{10}$. 


\begin{tabular}{|c|c|c|c|c|}
\hline & $\begin{array}{c}\text { Vertical } \\
\text { Coherent }\end{array}$ & $\begin{array}{c}\text { Vertical } \\
\text { Incoherent }\end{array}$ & $\begin{array}{c}\text { Horizontal } \\
\text { Coherent }\end{array}$ & $\begin{array}{c}\text { Horizontal } \\
\text { Incoherent }\end{array}$ \\
\hline Electric Field & -0.180 & -0.0595 & -0.0010 & +0.0572 \\
\hline DC Magnetic Field & -0.0111 & -0.0111 & +0.0107 & +0.0107 \\
\hline $\begin{array}{c}\text { Longitudinal } \\
\text { AC Magnetic Field }\end{array}$ & +0.143 & +0.0474 & +0.0008 & -0.0045 \\
\hline Betatron AC Magnetic & +0.0231 & & +0.013 & \\
\hline Direct Space Charge & & -0.0630 & & -0.0630 \\
\hline \hline Total Tune Shift & -0.0245 & -0.0862 & +0.0216 & -0.0407 \\
\hline
\end{tabular}

Table 3: Coherent and incoherent tune shifts in the Main Injector at $8.9 \mathrm{GeV}$. 


\section{RF Cavities and Coupled Bunch Instabilities}

Higher order cavity modes in the RF accelerating cavities are often the cause of coupled bunch instabilities. In this section we estimate the growth times of these instabilities based on measurements of the longitudinal impedances of the cavities and calculations of the transverse impedances. We also compare the estimates for the Main Injector to estimates of growth times in the Main Ring.

The RF cavities to be used for the Main Injector are the $18 \mathrm{RF}$ cavities presently installed and operating in the Main Ring. Stretched wire measurements of the longitudinal impedances of the cavities have been made [7] and the 6 modes with the largest impedances are listed in Table 4 . The cavity mode at $128 \mathrm{MHz}$ has limited the Main Ring performance in the past so a passive mode damper was designed and installed to reduce the shunt impedance of that particular mode [8] by a factor of 25 to the value of $6.3 \mathrm{k} \Omega / \mathrm{m}$ listed in Table 4 . This helped alleviate the problems with the coupled bunch instability.

The transverse modes of the RF cavities have been estimated previously using the computer code URMEL $[9,10]$ and the cavity modes with the 5 largest transverse impedances are listed in Table 5.

The standard perturbation theory, which neglects Landau damping effects, predicts that about half of the coupled bunch modes will have a positive growth rate associated with each higher order cavity mode. Therefore it is important to make some estimations on the effectiveness of Landau damping in preventing instability. We do this by comparing calculated growth 


\begin{tabular}{|c|c|c|c|c|c|}
\hline \multicolumn{2}{|c|}{ Longitudinal Coupled Bunch Growth Times (msec) } \\
\hline Freq of & $Z_{||}(\mathrm{k} \Omega)$ & \multicolumn{2}{|c|}{ dipole } & \multicolumn{2}{c|}{ quadrupole } \\
\cline { 3 - 6 } mode $(\mathrm{MHz})$ & per cavity & MI & MR & MI & MR \\
\hline 71.0 & 10.0 & 20.2 & 50.9 & 293 & 483 \\
100.0 & 2.5 & 65.5 & 177 & 461 & 797 \\
128.0 & 6.3 & 24.2 & 71.6 & 98.7 & 182 \\
223.0 & 111.0 & 2.1 & 10.3 & 2.1 & 5.5 \\
600.0 & 277.0 & 9.1 & 32.7 & 3.5 & 20.8 \\
850.0 & 49.0 & 91.5 & 350 & 45 & 241 \\
\hline
\end{tabular}

Table 4: Calculated growth times for the longitudinal dipole and quadrupole coupled bunch instability in the Main Injector and Main Ring with $18 \mathrm{RF}$ cavities. 


\begin{tabular}{|c|c|c|c|c|c|}
\hline \multicolumn{3}{|c|}{ Transverse Coupled Bunch Growth Times (msec) } \\
\hline Freq of & $Z_{\perp}(\mathrm{M} \Omega / \mathrm{m})$ & \multicolumn{2}{|c|}{ dipole } & \multicolumn{2}{|c|}{ quadrupole } \\
\cline { 3 - 6 } mode $(\mathrm{MHz})$ & per cavity & MI & MR & MI & MR \\
\hline 398 & 3.3 & 4.0 & 6.8 & 4.2 & 6.6 \\
454 & 1.9 & 8.1 & 13 & 8.0 & 13.4 \\
566 & 0.75 & 25.0 & 42 & 26 & 42 \\
1270 & 1.7 & 25 & 41 & 25 & 48 \\
1290 & 2.4 & 18.0 & 30 & 18.1 & 29.9 \\
\hline
\end{tabular}

Table 5: Calculated growth times for the transverse dipole and quadrupole coupled bunch modes in the Main Injector and Main Ring with $18 \mathrm{RF}$ cavities.

times in the Main Injector to the calculated growth times in the Main Ring. Since these cavities are presently used in the Main Ring, we can compare the calculated growth times to any observed instabilities.

The theoretical growth rate is derived by a perturbation expansion of the Vlasov Equations [11, 12]. The growth rate, without including Landau damping effects, is found by solving the matrix equation below for the value of the mode oscillation frequency $\Omega$,

$$
\begin{aligned}
& \left(\Omega-m \omega_{s}\right) \sigma_{m}(l)= \\
& \quad \sum_{p=-\infty}^{\infty} j \frac{2 \pi \omega_{s} I_{b} M m}{(p M+s) \omega_{o}^{2} h V \cos \phi_{s}} Z_{\|}\left[(p M+s) \omega_{o}\right] \sigma_{m}(p) \\
& \left.\quad \int_{0}^{\infty} J_{m}\left[(p M+s) \omega_{o} r\right] J_{m}\left[(l M+s) \omega_{o} r\right] \frac{\partial g_{o}(r)}{\partial r} d r, \quad \text { (for all } l\right)
\end{aligned}
$$


where $\operatorname{Im}\{\Omega\}<0$ implies an instability with an exponential growth time of $1 / \operatorname{Im}\{\Omega\}$. In this equation, $\omega_{o} / 2 \pi$ is the revolution frequency, $\omega_{s} / 2 \pi$ the synchrotron frequency, $I_{b}$ the average current per bunch, $M$ the number of bunches, $h$ the RF harmonic number, $V$ the peak RF voltage, $\phi_{s}$ the synchronous RF phase, $E$ the beam energy,

$s$ the coupled bunch mode number (ranging from 0 to $M-1$ ), $J_{m}$ the Bessel function of the first kind, and $r$ the distance from the center of the normalized longitudinal phase space.

Also $g_{o}(r)$ is the stationary longitudinal phase space distribution, $m$ refers to the single-bunch number in the longitudinal phase space $(m=1$ is the dipole mode, $m=2$ is the quadrupole mode, etc.), and $\sigma_{m}(l)$ are amplitudes of the beam spectrum at frequencies $\omega=(l M+s) \omega_{0}$.

Considering a high- $Q$ resonator impedance, such as an $R F$ cavity mode, a single term dominates in the sum over $p$ and the matrix Equation 12 reduces to

$$
\operatorname{Im}\{\Omega\}=\frac{-n \omega_{s} I_{b} M m}{2 h V \cos \phi_{s}} \operatorname{Re}\left\{Z_{\|}\left(n \omega_{o}\right)\right\} F_{m}
$$

where $\operatorname{Im}\{\Omega\}<0$ implies an instability, $n$ is the harmonic number nearest to the frequency of the high- $Q$ resonator, and $F_{m}$ is a form factor which depends 
on the bunch length and shape.

To calculate the form factor $F_{m}$ we use an elliptical density distribution longitudinal phase space of the form

$$
g_{o}(r)=\frac{3}{2 \pi\left(\tau_{L} / 2\right)^{2}}\left[1-\frac{r^{2}}{\left(\tau_{L} / 2\right)^{2}}\right]^{\frac{1}{2}}
$$

corresponding to a parabolic line charge density distribution

$$
\lambda(\tau)=\frac{3}{4\left(\tau_{L} / 2\right)}\left[1-\frac{\tau^{2}}{\left(\tau_{L} / 2\right)^{2}}\right]
$$

where the parameter $\tau_{L}$ is the full bunch length. For this distribution the form factor is

$$
F_{m}\left(x_{L}\right)=\frac{6}{x_{L}^{2}} \int_{0}^{\pi / 2} J_{m}^{2}\left(x_{L} \sin \theta\right) \sin \theta d \theta
$$

where $x_{L}=n \omega_{0} \tau_{L} / 2$. A plot of $F_{m}\left(x_{L}\right)$ for various $m$ values is shown in Figure 2.

In the Main Injector we find that the longitudinal coupled bunch growth times are shortest at $150 \mathrm{GeV}$. Therefore the growth times listed in Figure 4 are at $150 \mathrm{GeV}$ and use $M=498$ bunches with $N=6 \times 10^{10}$ per bunch (current per bunch $I_{b}=0.876 \mu \mathrm{A}$ ). With a $95 \%$ normalized longitudinal emittance of $0.25 \mathrm{eV}$-s and an RF voltage of $V=400 \mathrm{kV}$ the bunch length is $\tau_{L}=3.39 \mathrm{~ns}$. As a comparison we also calculate the growth times in the Main Ring with $M=1008$ bunches of $N=3 \times 10^{10}$ particles per bunch and a longitudinal emittance of $0.25 \mathrm{eV}$-s. The growth times for both machines are listed in Table 4.

Some of the calculated growth times for the Main Injector are short. It must be remembered however that these calculations do not include the 


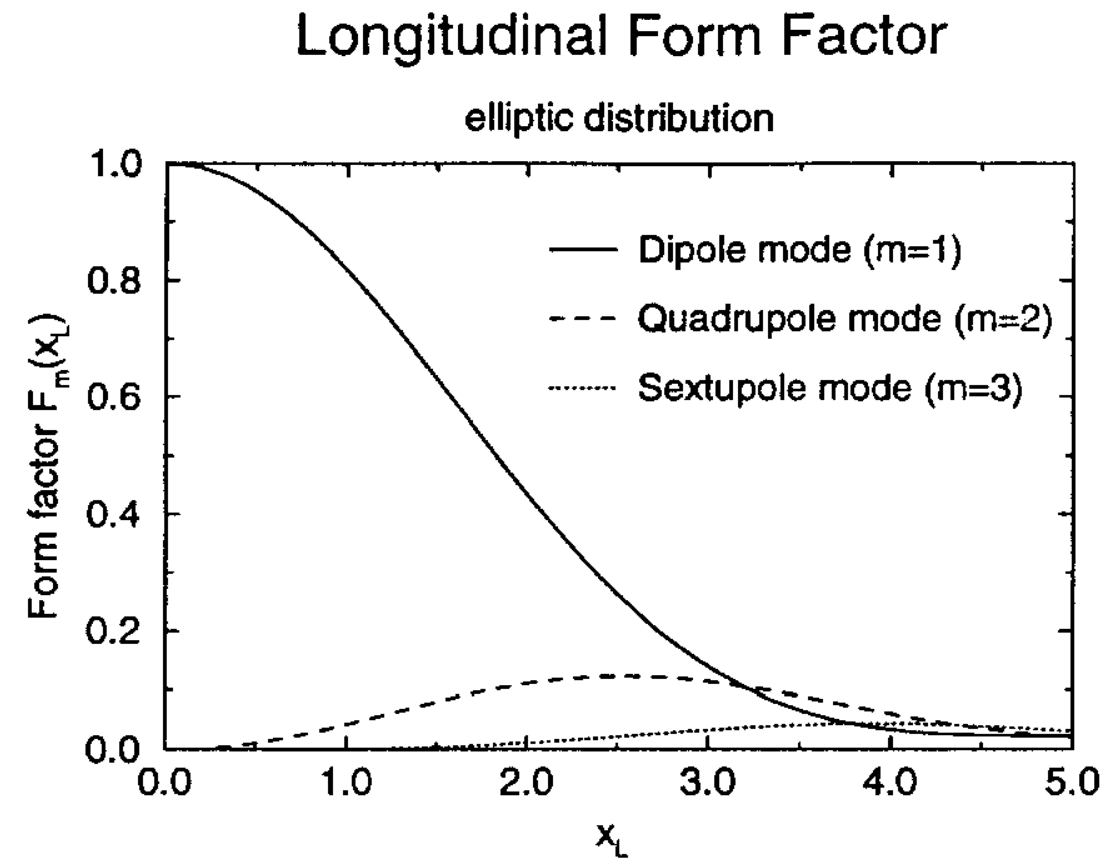

Figure 2: Longitudinal form factor for elliptical phase space density distribution.

Landau damping effect which may stabilize the beam and help prevent instability. The calculations are also worst case scenarios since it was assumed that the frequencies of the higher order modes in all 18 RF cavities are identical. In practice, the higher order modes are at slightly different frequencies in the individual cavities. Although the growth times are short, they are not alarming when compared to the calculated Main Ring growth times which are also short.

Experience with the Main Ring has shown that the $128 \mathrm{MHz}$ mode can sometimes be a problem in fixed target operation with a full ring of bunches. Also of concern is the short growth time of the $223 \mathrm{MHz}$ mode. As a result a set of passive mode dampers for the cavities was designed to reduce the shunt. impedance of the $223 \mathrm{MHz}$ mode and further damp the $128 \mathrm{MHz}$ mode as 
well. With this mode damper installed the $223 \mathrm{MHz}$ impedance is lowered to a value of about $10 \mathrm{k} \Omega$ reducing the calculated growth rate of the $223 \mathrm{MHz}$ mode by a factor of about 10 . If needed other RF cavity modes could be damped as well.

Also of concern is the coupled bunch instability with the short batches used in coalescing. Presently in the Main Ring there is evidence of an instability for short batches (about 11 consecutive bunches) with $3 \times 10^{10}$ particles per bunch. Since this type of instability depends on $R / Q$ and not on $R$, adding passive dampers does not remove the instability. The Main Injector calls for $6 \times 10^{10}$ particles per bunch so there could be substantial difficulty with short batch operation. Dealing with this problem will probably require the development of an active feedback damping system.

Calculated growth times for the transverse coupled bunch instability modes are based on the same perturbation approach used in the longitudinal case [11] and use the transverse impedances shown in Table 5. The growth rate for this instability, without including Landau damping effects, is found by solving the matrix equation for the mode oscillation frequency $\Omega$,

$$
\begin{aligned}
& \left(\Omega-m \omega_{s}\right) \sigma_{m}(l)= \\
& \sum_{p=-\infty}^{\infty} j \frac{e c I_{b} M}{2 E \nu_{\beta}} Z_{\perp}\left[\left(p M+s+\nu_{\beta}\right) \omega_{o}\right] \sigma_{m}(p) \\
& \int_{0}^{\infty} J_{m}\left[\left(p M+s+\nu_{\beta}\right) \omega_{o} r-\omega_{\xi} r\right] \\
& \left.\quad J_{m}\left[\left(l M+s+\nu_{\beta}\right) \omega_{o} r-\omega_{\xi} r\right] g_{o}(r) r d r, \quad \text { (for all } l\right)
\end{aligned}
$$

and $\operatorname{Im}\{\Omega\}<0$ implies an instability with exponential growth time $1 / \operatorname{Im}\{\Omega\}$. In Equation 17 the variables are the same as in the longitudinal case, $\nu_{\beta}=\nu_{\mathrm{H}}$ 
or $\nu_{\mathrm{V}}$ is the tune of the machine, and $\omega_{\xi}=\omega_{o} \xi / \eta$ accounts for chromaticity effects. The $\sigma_{m}(l)$ are the amplitudes of the transverse beam spectrum at frequencies of $\omega=\left(l M+s+\nu_{\beta}\right) \omega_{o}-\omega_{\xi}$. Here $\eta=\gamma_{t}^{-2}-\gamma^{-2}$ is the frequency slip parameter, where $\gamma_{t}=20.4$ is the transition gamma.

For the case of a high- $Q$ resonator impedance a single term dominates in the sum over $p$ and Equation 17 reduces to

$$
\operatorname{Im}\{\Omega\}=-\frac{e I_{b} M}{4 \pi E \nu_{\beta}} \operatorname{Re}\left\{Z_{\perp}\left[\left(n-\nu_{\beta}\right) \omega_{o}\right]\right\} F_{m}(\alpha) .
$$

where $\alpha=\left[\left(n+\nu_{\beta}\right) \omega_{o}-\omega_{\xi}\right] \tau_{L} / 2, F_{m}(\alpha)$ is a form factor that depends on the longitudinal distribution of the bunches, and $n$ is the positive harmonic nearest to the high- $\mathrm{Q}$ resonator.

For the elliptical phase space density distribution of Equation 14 the form factor can be expressed as

$$
F_{m}(\alpha)=2 \pi \int_{0}^{\infty} J_{m}^{2}(\alpha x)\left(1-x^{2}\right)^{\frac{1}{2}} x d x
$$

Figure 3 shows a plot of $F_{m}(\alpha)$ for various values of $m$.

The growth times for both the Main Injector and the Main Ring are calculated at an energy of $8.9 \mathrm{GeV}$, a $95 \%$ normalized longitudinal emittance of $0.1 \mathrm{eV}$-s, and an $\mathrm{RF}$ voltage $V=400 \mathrm{kV}$. The number of protons per bunch is $6 \times 10^{10}$ in the Main Injector $3 \times 10^{10}$ in the Main Ring and the results are listed in Table 5. The calculated growth times in the Main Injector are about one half of those in the Main Ring. So far there has not been any evidence observed of transverse coupled bunch modes in the Main Ring driven by the transverse RF cavity modes. This is due to the Landau damping given by the tune spread. 


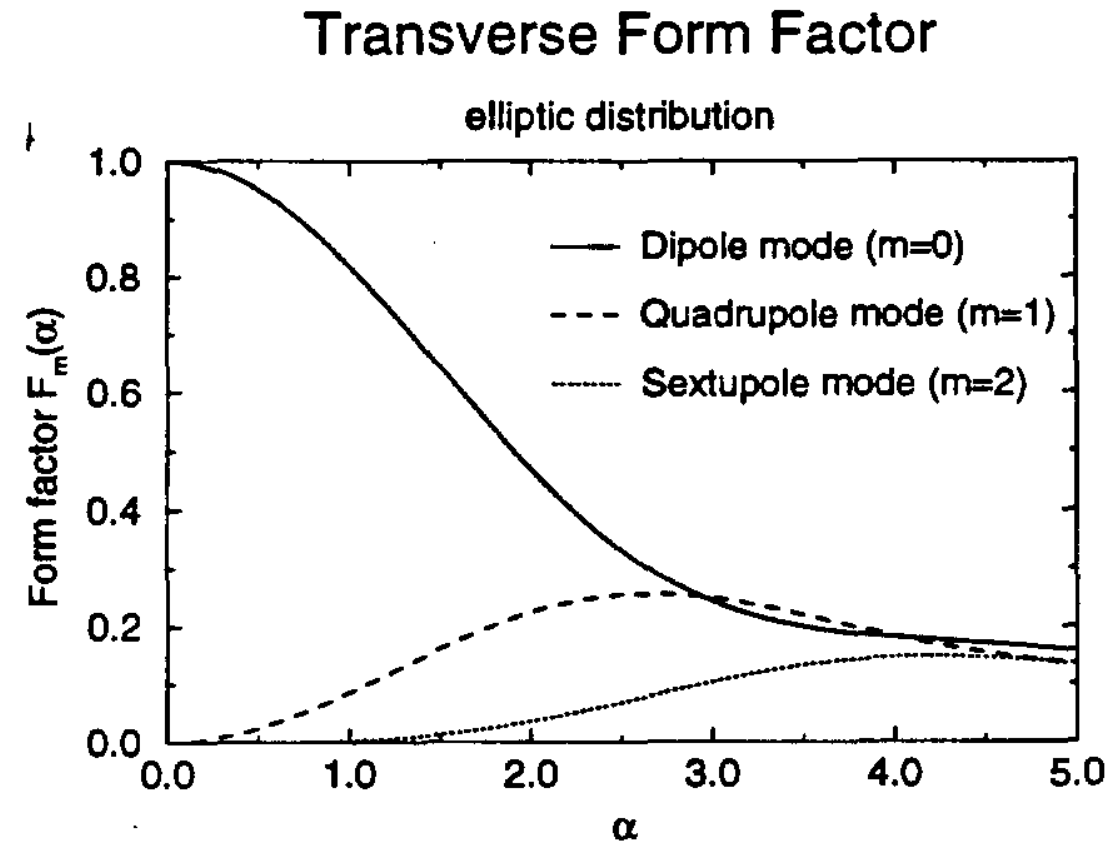

Figure 3: Transverse coupled bunch instability form factor as function of $\alpha$. (See Equation 19)

\section{Resistive Wall}

The shape of the Main Injector beampipe is shown in Figure 1. It is approximately elliptical with a full height of $5.31 \mathrm{~cm}(2.09$ inches) and a full width of $12.3 \mathrm{~cm}$ (4.84 inches). The beampipe is stainless steel, has a thickness of $1.5 \mathrm{~mm}$, and a resistivity of $\rho=74 \mu \Omega-\mathrm{cm}$. When installed in the Main Injector magnets and under vacuum the beampipe deforms reducing its height to $5.08 \mathrm{~cm}$. The shape of the beampipe makes an exact calculation of the resistive wall impedance difficult. Instead we make estimations using exact solutions for beampipes with circular [13] and rectangular [1] cross sections. We use a circular beampipe with a radius of $b=2.39 \mathrm{~cm}$ and a rectangular beampipe with a height of $4.78 \mathrm{~cm}$ and a width of $12.0 \mathrm{~cm}$. 
For a circular beampipe with beam traveling down its center the longitudinal resistive wall impedance is given by the expression

$$
Z_{\|}(\omega)=(1+j) \frac{R \rho}{b \delta}
$$

where

$$
\delta=\sqrt{\frac{2 \rho}{\mu_{\mathrm{o}} \omega}}
$$

is the skin depth, $R=528.3 \mathrm{~m}$ is the radius of the machine, and $b=2.39 \mathrm{~cm}$ is the radius of the beampipe. Similarly the transverse resistive wall impedance is given by

$$
Z_{\perp}(\omega)=(1+j) \frac{2 c R \rho}{\omega l^{3} \delta} .
$$

The expressions given above for the longitudinal and transverse impedances are valid only when the skin depth in the beampipe is less than the thickness of the beampipe. When the skin depth is greater than the thickness of the beampipe, then the thickness of the beampipe must be substituted for the skin depth in Equation 20 and 22 and the imaginary part vanishes.

Using the circular approximation to the Main Injector beampipe we get the results listed in Table 6 . The results are expressed as a function of the revolution harmonic number $n$ for both high and low frequencies.

In the interest of understanding the effect that the shape of the beam pipe has on the resistive wall instability, the beampipe cross section is also approximated by a rectangle. The full height and width of the rectangular beampipe is chosen to be $h=4.78 \mathrm{~cm}$ by $w=12.0 \mathrm{~cm}$ to match the inside diameters of the actual elliptical beampipe. The results can be expressed as 


\begin{tabular}{|c|c|c|}
\hline \multicolumn{3}{|c|}{ Circular Beampipe } \\
\hline & $n<n_{c}$ & $n>n_{\mathrm{c}}$ \\
\hline$Z_{\|} / n$ & $10.9 / n \Omega$ & $(1+j) 11.3 / \sqrt{n} \Omega$ \\
\hline$Z_{\perp}$ & $20.3 / n \mathrm{M} \Omega / \mathrm{m}$ & $(1+j) 21.1 / \sqrt{n} \mathrm{M} \Omega / \mathrm{m}$ \\
\hline
\end{tabular}

\begin{tabular}{|c|c|c|}
\hline \hline \multicolumn{3}{|c|}{ Rectangular Beampipe } \\
\hline & $n<n_{c}$ & $n>n_{c}$ \\
\hline$Z_{\|} / n$ & $10.8 / n \Omega$ & $(1+j) 11.2 / \sqrt{n} \Omega$ \\
\hline$Z_{\perp x}$ & $8.24 / n \mathrm{M} \Omega / \mathrm{m}$ & $(1+j) 8.55 / \sqrt{n} \mathrm{M} \Omega / \mathrm{m}$ \\
\hline$Z_{\perp y}$ & $16.7 / n \mathrm{M} \Omega / \mathrm{m}$ & $(1+j) 17.3 / \sqrt{n} \mathrm{M} \Omega / \mathrm{m}$ \\
\hline
\end{tabular}

Table 6: Resistive wall impedance as a function of revolution harmonic $n$ for circular beampipe with radius $b=2.39 \mathrm{~cm}$ and rectangular beampipe $4.78 \mathrm{~cm}$ by $12.0 \mathrm{~cm}$. Results are given for frequencies where the skin depth is less (greater) than the thickness of the beampipe $n>n_{c}\left(n<n_{c}\right)$ where $n_{c}=0.922$. 
the product of a form factor times the circular result with radius $b=h / 2$ [1]. For the width to height ratio 2.51 the form factors are found to be $F_{\| \mid}=0.993$, $F_{\perp x}=0.406$, and $F_{\perp y}=0.822$. Thus the longitudinal impedance does not differ much between the circular and rectangular case. The transverse impedance in the $y$ direction in the rectangular case is not much less than that in the circular approximation. However, the transverse impedance in the $x$ direction is only about one half of that in the $y$ direction.

The most important effect of the resistive wall instability is the transverse coupled bunch mode at low frequencies and low energies. The growth rate for this instability, without including Landau damping, is given in Equation 17.

At low frequencies the resistive wall impedance is proportional to $\omega^{-\frac{1}{2}}$ and therefore sharply peaked at the origin. Since the vertical tune is 25.4 the coupled bunch mode $s=-26$ has the largest growth rate. The lowest and most relevant frequency is only $-0.6 \omega_{o} / 2 \pi=54 \mathrm{kHz}$ which is very small. Since the transverse impedance is so sharply peaked at the origin we consider only the term with $p=0$ and $s=-26$ in Equation 17. This reduces the expression for the growth rate to

$$
\left.\operatorname{Im}\{\Omega\}=+\frac{e c I_{b} M}{4 \pi E \nu_{\beta}} \operatorname{Re}\left\{Z_{\perp}\left[\left(s+\nu_{\beta}\right) \omega_{o}\right)\right]\right\} F_{m}(\alpha)
$$

where $\alpha=\left[\left(n+\nu_{\beta}\right) \omega_{o}-\omega_{\xi}\right] \tau_{L} / 2$ and $\mathrm{F}_{m}(\alpha)$ is a form factor which depends on the bunch length and the chromaticity and, again, $\operatorname{Im}\{\Omega\}<0$ implies an instability. For the elliptical phase space density distribution in Equation 14 the form factor is given by Equation 19 and is plotted in Figure 3.

Using the low frequency estimates of the Lambertson magnets (see Section $\mathrm{H})$ and the beampipe resistive wall impedance we calculate the cor- 
responding growth time for mode $s=-26$. The resistive wall impedance of the beampipe is $\operatorname{Re}\left\{Z_{\perp}\left(-0.6 \omega_{0}\right)\right\}=-27.8 \mathrm{M} \Omega / \mathrm{m}$ and the Lambertson impedance is $\operatorname{Re}\left\{Z_{\perp}\left(-0.6 \omega_{0}\right)\right\}=-35 \mathrm{M} \Omega / \mathrm{m}$. We assume $6 \times 10^{10}$ particles per bunch $\left(M I_{b}=0.434 \mathrm{amps}\right)$, a $95 \%$ normalized longitudinal emittance of $0.1 \mathrm{eV}$-s and an RF voltage of $400 \mathrm{kV}$. With the chromaticity set to zero the form factor is unity $(\alpha=0)$ giving a calculated growth time of $0.35 \mathrm{~ms}$ at $8.9 \mathrm{GeV}$.

Althougl this is a fast growth time, some observations of the Main Ring [14] lead us to believe that the resistive wall instability will not be a problem. Using an impedance of $-98 \mathrm{M} \Omega / \mathrm{m}$ and intensity of $1.5 \times 10^{13} \mathrm{in}$ the Main Ring, the calculated growth time of the resistive wall instability is $0.65 \mathrm{~ms}$. However at this intensity beam is stable in the Main Ring without providing any method of damping (transverse dampers turned off, chromaticity set to zero, and no octupole magnets used). At the higher intensity of $2 \times 10^{13}$ beam is stable whenever any one of the following is true: 1) just the transverse dampers turned on, 2) just the chromaticity set between -30 to -40 , and 3) just the octupole magnets turned on. The Main Injector, in addition to the transverse damper, has all three methods available to provide further damping. For instance, changing the chromaticity of the Main Injector at injection from zero to -20 increases the growth time from $0.35 \mathrm{~ms}$ to 2 msec. Thus we should not expect any problems with the transverse resistive wall instabilities in the Main Injector. 


\section{E Beam Position Monitors}

The Main Injector BPM consists of 4 stripline pickups which are cut out of the approximately elliptical beampipe. Two pickups are located on each of the top and bottom surfaces of the beampipe and are spaced $40 \mathrm{~mm}$ apart from center to center as shown in Figure 1. Each stripline is about $1 \mathrm{~cm}$ wide, $l=40 \mathrm{~cm}$ long, and has a characteristic impedance of $50 \Omega$. The downstream end of each stripline is shorted while the upstream end is connected in parallel with a $50 \Omega$ cable leading to the $\mathrm{RF}$ module.

Image current traveling in the beampipe which enters the stripline at the upstream end will see the $Z_{c}=50 \Omega$ cable and the shorted $Z_{c}=50 \Omega$ stripline in parallel. Therefore the impedance of an individual pickup is given by the expression

$$
Z_{\mathrm{s}}=\frac{Z_{c}}{2}[1-\cos (2 k l)+j \sin (2 k l)]
$$

where $k=\omega / c$ is the wave number.

As a bunch passes the BPM only a fraction of the image current on the beampipe is picked up by the stripline. To estimate this fraction, the beampipe is approximated as an elliptical cylinder with $5.12 \mathrm{~cm}$ by $12.30 \mathrm{~cm}$ diameter. The charge density induced on an elliptical conductor by a line charge at the center was then calculated using the program POISSON [15] and the assumption made that the fraction of image current entering the stripline pickup is the same as the fraction of static charge density induced on the stripline which is valid when $\omega<\gamma c / d$ where $d$ is the distance from the beam to the stripline. For the model used in this paper, the fraction is $f=0.055$ for each stripline. 
The beam impedance due to a single stripline is then calculated by noting that the power lost in the stripline must equal the power lost by the beam. The beam impedance for a single stripline is then $f^{2} Z_{\mathrm{s}}$ or $Z=4 f^{2} Z_{\mathrm{s}}$ for an entire BPM. With $N=208 \mathrm{BPMs}$ in the Main Injector the net longitudinal impedance is then

$$
Z_{\|}=2 N f^{2} Z_{c}[1-\cos (2 k l)+j \sin (2 k l)] .
$$

To estimate the transverse impedances, an approach similar to the longitudinal case is used. A dipole current at the center of the BPM induces image current in the beampipe and a certain amount of the current enters the striplines. For a dipole current density in the $y$ direction of magnitude $1 \mathrm{amp}-\mathrm{mm}$ the dipole current entering the stripline is equal to 2.8 amps. With this information the longitudinal impedance for the dipole mode is calculated to be $Z_{1 \| y}=31.1 Z_{\mathrm{s}} \Omega / \mathrm{m}^{2}$ for a single BPM and $Z_{1 \| y}=6470 Z_{\mathrm{s}} \Omega / \mathrm{m}^{2}$ for all 208 BPMs. Using the Panofsky-Wenzel theorem [16] the transverse impedance is given by the relation $Z_{\perp y}=\frac{c}{\omega} Z_{1 \| y}$.

In the $x$ direction, with a dipole current of $1 \mathrm{amp}-\mathrm{mm}$ at the center of the beampipe, the current entering the stripline is equal to 2.0 amps giving a longitudinal impedance for the dipole mode of $Z_{1 \| x}=16.1 Z_{\mathrm{s}} \Omega / \mathrm{m}^{2}$ for a single BPM and $Z_{1 \| x}=3350 Z_{\mathrm{s}} \Omega / \mathrm{m}^{2}$ for all 208 BPMs. Thus the transverse impedance is calculated to be $Z_{\perp x}=\frac{c}{\omega} Z_{1|| x}$.

In the low frequency limit, the total longitudinal impedance of all 208 BPMs becomes $Z_{\|} / n=j 0.095 \Omega$ and the total transverse impedances becomes $Z_{\perp y}=j 5.15 \mathrm{k} \Omega / \mathrm{m}$ and $Z_{\perp x}=j 2.66 \mathrm{k} \Omega / \mathrm{m}$. These impedances are much lower than other components in the ring. This is mainly due to the 
relatively narrow width of the stripline pickups. Thus the BPMs are not expected to be a problem.

\section{F Bellows}

The beampipe bellows will consist of approximately 10 to 17 convolutions extending over one inch. The inner dimensions of the bellows are an ellipse of similar size to the beampipe and the convolutions extend $1.28 \mathrm{~cm}$ ( 0.5 inches). To estimate the impedance the elliptical shape was approximated by a circular beampipe with radius $2.67 \mathrm{~cm}$ (1.05 inches). This was done for both 10 and 17 bellow convolutions using the program TBCI [17]. The calculated longitudinal impedance of the 10 and 17 bellow convolution models are very similar. The real part of the longitudinal and transverse impedance of a single bellow with 10 convolutions are shown in Figures 4 and 5 .

The longitudinal impedances of a single bellow can be characterized approximately as two resonators of the form

$$
Z_{\|}(\omega)=\frac{R_{s}}{1+j Q\left(\omega / \omega,-\omega_{r} / \omega\right)}
$$

with values $R_{s}=400 \Omega, Q=5.3, \omega_{r} / 2 \pi=4 \mathrm{GHz}$ and $R_{s}=120 \Omega, Q=4.3$, and $\omega_{r} / 2 \pi=8.5 \mathrm{GHz}$.

In the Main Injector there are 516 bellows giving a total longitudinal impedance of $R_{s} / n=4.66 \Omega$ or $R_{s} / Q=38.9 \mathrm{k} \Omega$ at $4 \mathrm{GHz}$ and $R_{s} / n=$ $0.658 \Omega$ or $R_{s} / Q=14.4 \mathrm{k} \Omega$ at $8.5 \mathrm{GHz}$. The typical rms bunch length at $8.9(150) \mathrm{GeV}$ is $1.24(0.69) \mathrm{ns}$ corresponding to a rms spectral spread of $0.13(0.23) \mathrm{GHz}$. On the other hand, the widths of the bellows impedance 


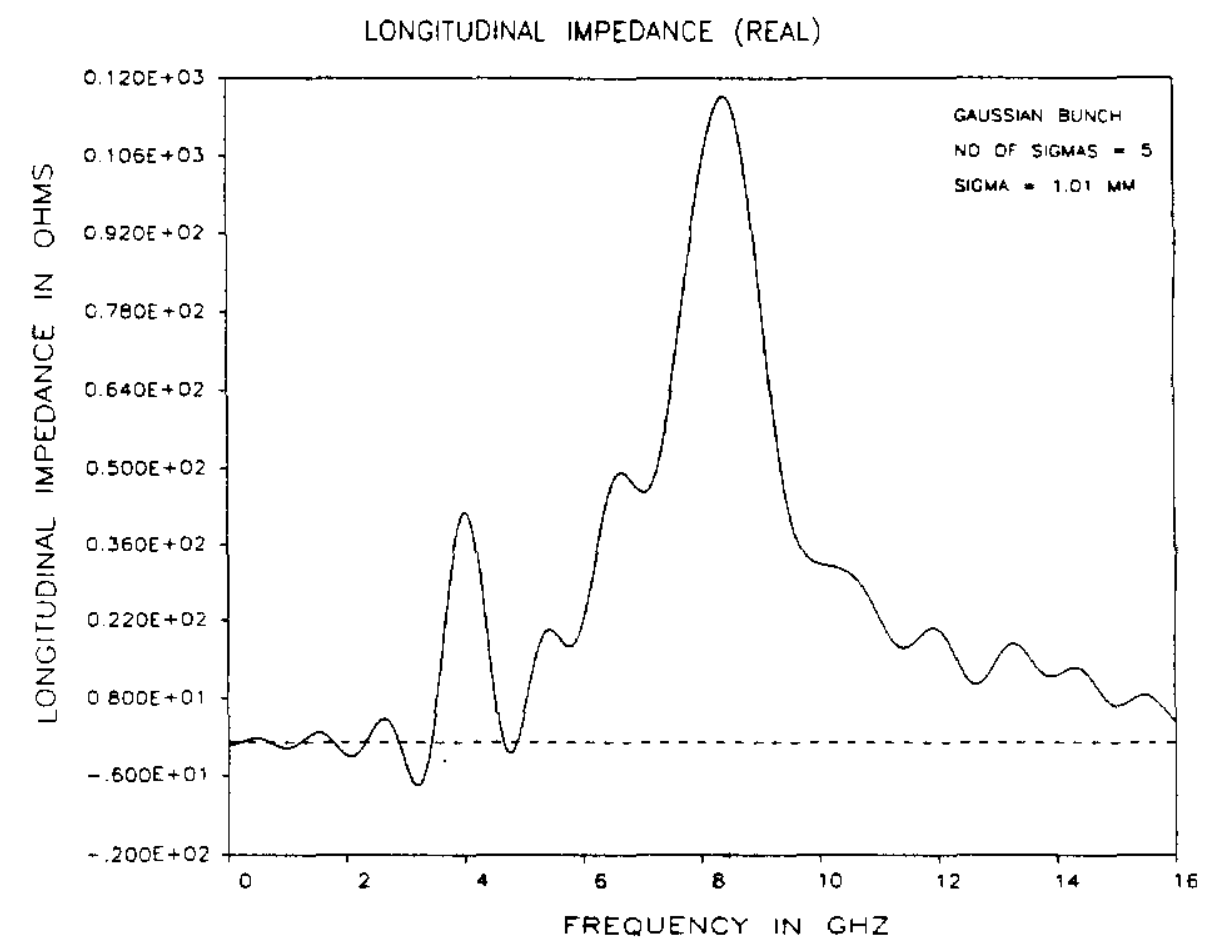

Figure 4: Real part of the longitudinal impedance for the circular bellows with 10 convolutions.

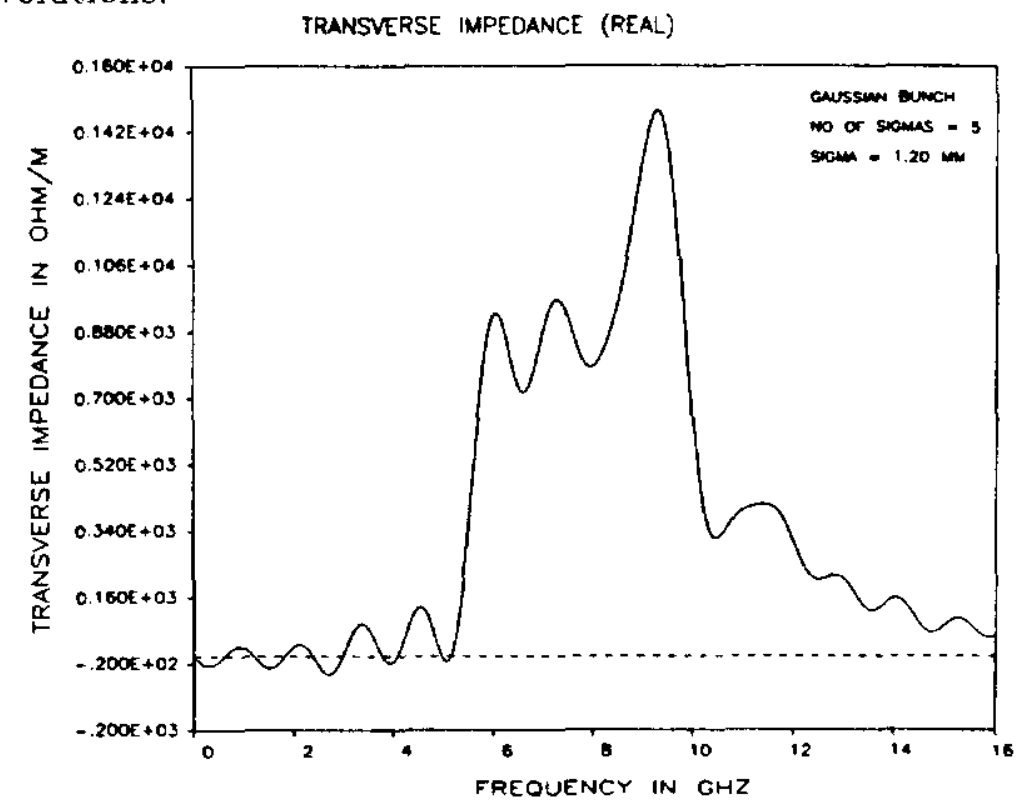

Figure 5: Real part of the transverse impedance for the circular bellows with 10 convolutions. 
resonances are $0.75 \mathrm{GHz}$ at and $2.0 \mathrm{GHz}$ the resonance frequencies of $4 \mathrm{GHz}$ and $8.5 \mathrm{GHz}$. Therefore these resonances should be treated as broadband and the impedance values should be compared with the microwave instability budget limits as calculated in Section $\mathrm{I}$, which gives $\left|Z_{\|} / n\right|<36.9 \Omega$ at $8.9 \mathrm{Gev}$ and $\left|Z_{\|} / n\right|<6.6 \Omega$ at $150 \mathrm{Gev}$.

The transverse impedance of the bellows can be approximated as a resonator of the form

$$
Z_{\perp}(\omega)=\frac{1}{\omega} \frac{R_{\perp} \omega_{r}}{1+j Q\left(\omega / \omega_{r}-\omega_{r} / \omega\right)}
$$

with values $R_{\perp}=1.5 \mathrm{k} \Omega / \mathrm{m}, Q=5.3$, and $\omega_{r} / 2 \pi=8 \mathrm{GHz}$. With 516 bellows in the Main Injector the total transverse impedance is then $R_{\perp} / n=$ $8.74 \mathrm{k} \Omega / \mathrm{m}$ and $R_{\perp} / Q=146 \mathrm{k} \Omega / \mathrm{m}$ at $8 \mathrm{GHz}$. Both of these values are well below the microwave instability limits of $\left|Z_{\perp} / n\right|<55 \mathrm{k} \Omega / \mathrm{m}$ and $R_{\perp}<$ $71.6 \mathrm{M} \Omega / \mathrm{m}$ calculated in Section I at $8.9 \mathrm{GeV}$. Therefore the transverse impedance of the bellows should not create any problems in the Main Injector.

\section{G Vacuum Valves}

Although the vacuum valve design is not yet completed, its general shape is known. In this section we consider the two different shapes shown in Figures 6 and 7. The difference between the two designs is the presence or absence of the cylindrical pillbox cavities on either side of the valve. Using these shapes the 3D computer code MAFIA [18] was used to calculate the longitudinal and transverse impedances. The beampipe cross section is approximated by an ellipse with $5.12 \mathrm{~cm}$ by $12.3 \mathrm{~cm}$ diameters. The resistivity of the vacuum 


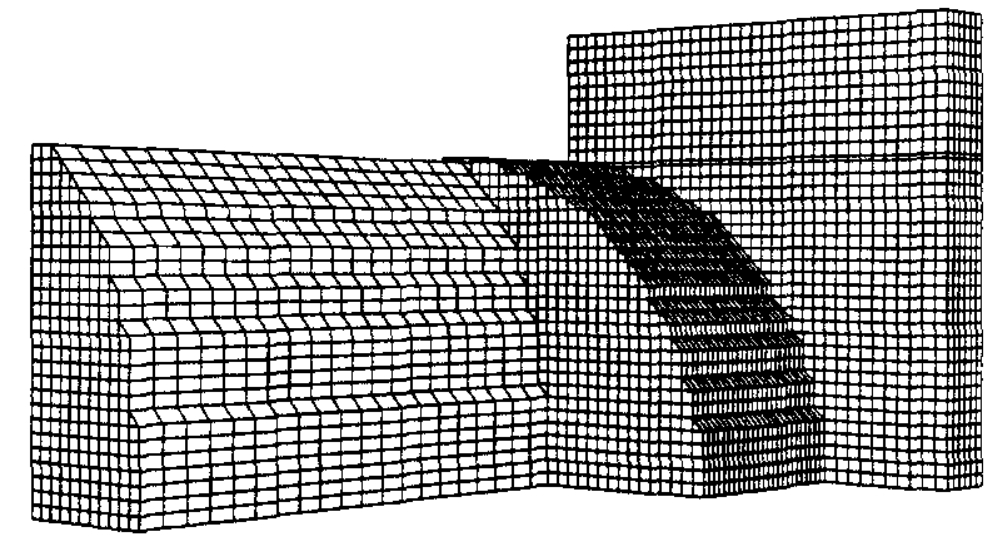

Figure 6: Approximate geometry of Main Injector vacuum valve with pillbox cavities.

valve is the same as the beampipe, $\rho=74 \mu \Omega-\mathrm{cm}$, and the results from MAFIA are converted to match this assumption. The impedances of a single vacuum valve are shown in Table 7 and the net impedances of all 30 valves in the Main Injector are shown in Table 8. The impedances are assumed to have impedances of the forms in Equation 26 and 27.

Calculations for the coupled bunch growth rates show that the vacuum valve modes are benign. This is mainly due to the fact that the modes in the valves are at frequencies above the beam spectrum frequencies. All modes have a growth time of greater than $100 \mathrm{~ms}$ except for the quadrupole mode of the $2.46 \mathrm{GHz}$ mode which has a growth rate of $62 \mathrm{~ms}$.

Also of concern is the potential microwave instability due to the vacuum valves. Section I discusses the threshold values for resonator impedances. 


\begin{tabular}{|c|c|c|}
\hline \multicolumn{3}{|c|}{ Vacuum valve with pillbox cavities } \\
\hline Freq $(\mathrm{GHz})$ & $Z_{\|}(\mathrm{k} \Omega)$ & $Q$ \\
\hline 1.511 & 76 & 2090 \\
2.460 & 117 & 3800 \\
\hline \hline Freq $(\mathrm{GHz})$ & $Z_{\perp x}(\mathrm{M} \Omega / \mathrm{m})$ & $Q$ \\
\hline 1.52 & 1.7 & 3000 \\
3.11 & 0.071 & 4000 \\
\hline \hline Freq $(\mathrm{GHz})$ & $Z_{\perp y}(\mathrm{M} \Omega / \mathrm{m})$ & $Q$ \\
\hline 1.928 & 0.47 & 1980 \\
3.154 & 0.21 & 3810 \\
\hline
\end{tabular}

\begin{tabular}{|c|c|c|}
\hline \multicolumn{3}{|c|}{ Vacuum valve without pillbox cavities } \\
\hline Freq $(\mathrm{GHz})$ & $Z_{\|}(\mathrm{k} \Omega)$ & $Q$ \\
\hline 1.30 & 52 & 1600 \\
2.85 & 13.4 & 2700 \\
\hline \hline Freq $(\mathrm{GHz})$ & $Z_{\perp x}(\mathrm{M} \Omega / \mathrm{m})$ & $Q$ \\
\hline 2.95 & 0.422 & 2500 \\
\hline \hline Freq $(\mathrm{GHz})$ & $Z_{\perp y}(\mathrm{M} \Omega / \mathrm{m})$ & $Q$ \\
\hline 3.10 & 0.032 & 2500 \\
\hline
\end{tabular}

Table 7: Estimated frequency, impedance per vacuum valve, and $Q$ value of longitudinal and transverse modes in two different Main Injector vacuum valve designs. See Figures 6 and 7 . 


\begin{tabular}{|c|c|c|}
\hline \multicolumn{3}{|c|}{ Vacuum valve with pillbox cavities } \\
(See Figure 6$)$ \\
\hline Freq $(\mathrm{GHz})$ & $\left|Z_{\|} / n\right|(\Omega)$ & $R_{\|} / Q(\Omega)$ \\
\hline 1.511 & 135 & 1090 \\
2.460 & 129 & 924 \\
\hline \hline Freq $(\mathrm{GHz})$ & $\left|Z_{\perp x} / n\right|(\mathrm{k} \Omega / \mathrm{m})$ & $R_{\perp_{x}} / Q(\mathrm{k} \Omega / \mathrm{m})$ \\
\hline 1.52 & 3.0 & 17 \\
3.11 & 0.06 & 0.53 \\
\hline \hline Freq $(\mathrm{GHz})$ & $\left|Z_{\perp_{y}} / n\right|(\mathrm{k} \Omega / \mathrm{m})$ & $R_{\perp_{y}} / Q(\mathrm{k} \Omega / \mathrm{m})$ \\
\hline 1.928 & .650 & 7.2 \\
3.154 & .178 & 1.6 \\
\hline
\end{tabular}

\begin{tabular}{|c|c|c|}
\hline \multicolumn{3}{|c|}{ Vacuum valve without pillbox cavities } \\
(See Figure 7) \\
\hline Freq $(\mathrm{GHz})$ & $\left|Z_{\|} / n\right|(\Omega)$ & $R_{\|} / Q(\Omega)$ \\
\hline 1.30 & 108 & 975 \\
2.85 & 12.6 & 150 \\
\hline \hline Freq $(\mathrm{GHz})$ & $\left|Z_{\perp x} / n\right|(\mathrm{k} \Omega / \mathrm{m})$ & $R_{\perp x} / Q(\mathrm{k} \Omega / \mathrm{m})$ \\
\hline 2.95 & .387 & 5.04 \\
\hline \hline Freq $(\mathrm{GHz})$ & $\left|Z_{\perp y} / n\right|(\mathrm{k} \Omega / \mathrm{m})$ & $R_{\perp y} / Q(\mathrm{k} \Omega / \mathrm{m})$ \\
\hline 3.10 & 0.028 & 0.384 \\
\hline
\end{tabular}

Table 8: Estimated net impedances of 30 vacuum valves in the Main Injector. 


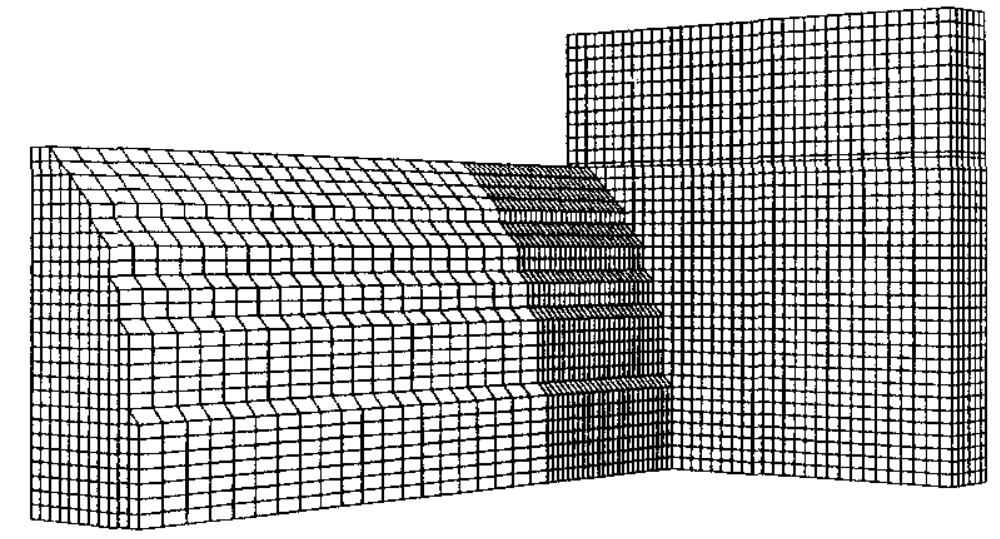

Figure 7: Approximate geometry of Main Injector vacuum valve without pillbox cavities.

The conclusion is that the vacuum valves must have a total longitudinal impedance of their resonator modes $R_{\|} / Q \leq 26.7 \mathrm{k} \Omega$ and their total transverse impedance $R_{\perp} / Q \leq 71.6 \mathrm{~N} \Omega / \mathrm{m}$. The vacuum valve impedances are less than these threshold values.

\section{H Lambertsons}

The main concern of the Lambertson magnets is the low frequency component created by the exposure of the bare magnet laminations to beam. A rough estimation of the Lambertson magnets is made by approximating the magnet as a series of annular laminations of $0.953 \mathrm{~mm}$ width. The inner radius is chosen to be $b=2.54 \mathrm{~cm}$ and the outer radius is chosen to be $d=5.08 \mathrm{~cm}$. 
The low frequency current traveling through the magnet is assumed to flow in one lamination from the inner radius to the outer radius, cross over to the next lamination, and flow from the outer radius to the inner radius. Even though we are concerned about the low frequency impedance, the skin depth is less than the lamination thickness at the frequencies we are considering. The current is therefore constrained to one skin depth in the laminations. The impedance of the magnet is then calculated by adding up the resistance along the entire current path.

For the current traveling from the inner radius to the outer radius the net impedance is

$$
Z=(1+j) \frac{\rho}{\pi \delta} \ln \left(1+\frac{d}{b}\right)
$$

where $\rho$ is the resistivity of the laminatjons and $\delta$ is the skin depth. For the current traveling along the inner tip of the laminations the resistance per unit length is

$$
Z=(1+j) \frac{\rho}{2 \pi b \delta}
$$

The lamination material has a resistivity of $\rho=20 \mu \Omega-\mathrm{cm}$ and a relative permeability of $\mu=100$. With 25.8 meters of Lambertson magnets in the Main Injector the total estimated low frequency impedance is

$$
Z_{\|} / n=(1+j) \frac{16.4}{\sqrt{n}} \mathrm{M} \Omega
$$

To estimate the transverse impedance we use the approximate relation

$$
Z_{\perp}=\frac{2 c}{b^{2}} \frac{Z_{\|}}{\omega}
$$


and arrive at

$$
Z_{\perp}=(1+j) \frac{26.8}{\sqrt{n}} \mathrm{M} \Omega / \mathrm{m}
$$

It should be noted that the Lambertson magnet was assumed to have a circular geometry with inner radius of $b=2.54 \mathrm{~cm}$. The actual shape of the Lambertson is much different so this estimate can only be approximate. Using a slightly larger inner radius can change the impedance by a significant amount (i.e. if $b$ is $10 \%$ larger the longitudinal impedance drops by $10 \%$ and the transverse impedance drops by $25 \%$ ).

The impedance at higher frequencies was also calculated assuming the gap between the laminations acts as a radial waveguide. The material in the gap has a relative permittivity $\epsilon=6$ and a small conductivity $\sigma=0.01 \Omega^{-1} \mathrm{~m}^{-1}$. The results of this type of analysis are shown in Figures 8 and 9.

\section{Microwave Instabilities}

The possibility of microwave instability is an important consideration for the Main Injector and in this section we calculate the microwave instability impedance budget. Although particularly relevant at transition, this paper does not consider transition crossing issues. This is, however, an important aspect of the Main Injector and deserves further attention.

The microwave stability limit for bunched beam with a Gaussian momentum distribution in the presence of a broadband resonator [19] can be expressed in terms of the rms momentum spread of a bunch $\sigma_{p} / p$ and the 


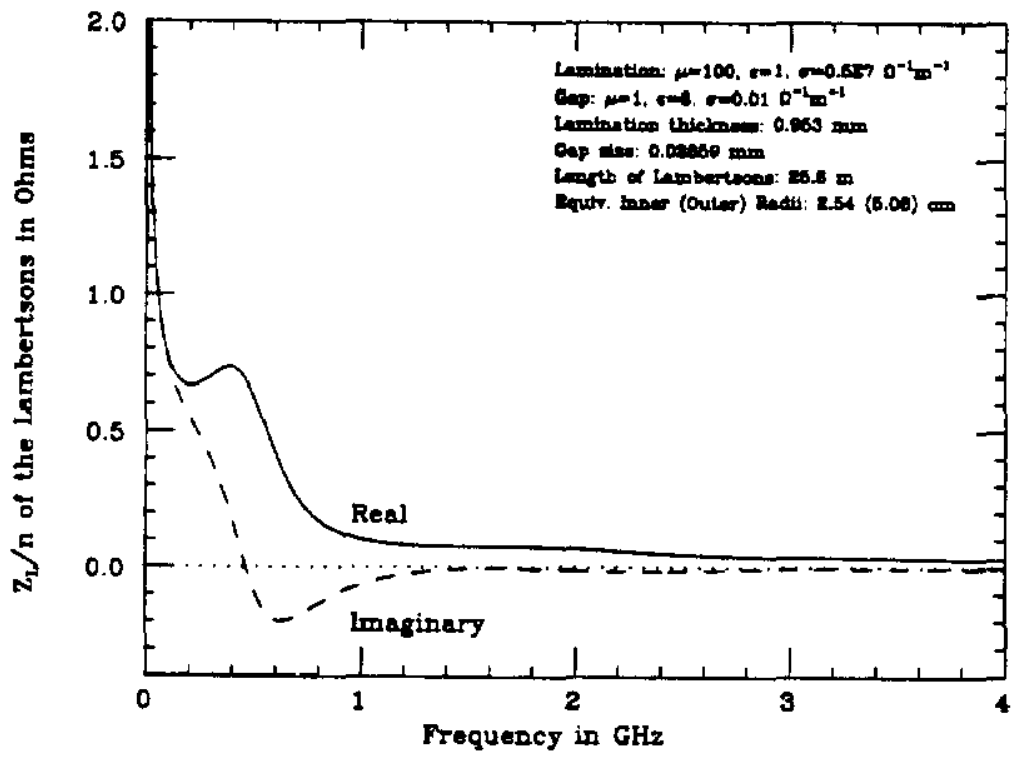

Figure 8: Real and imaginary part of the longitudinal impedance for the Lambertson magnets.

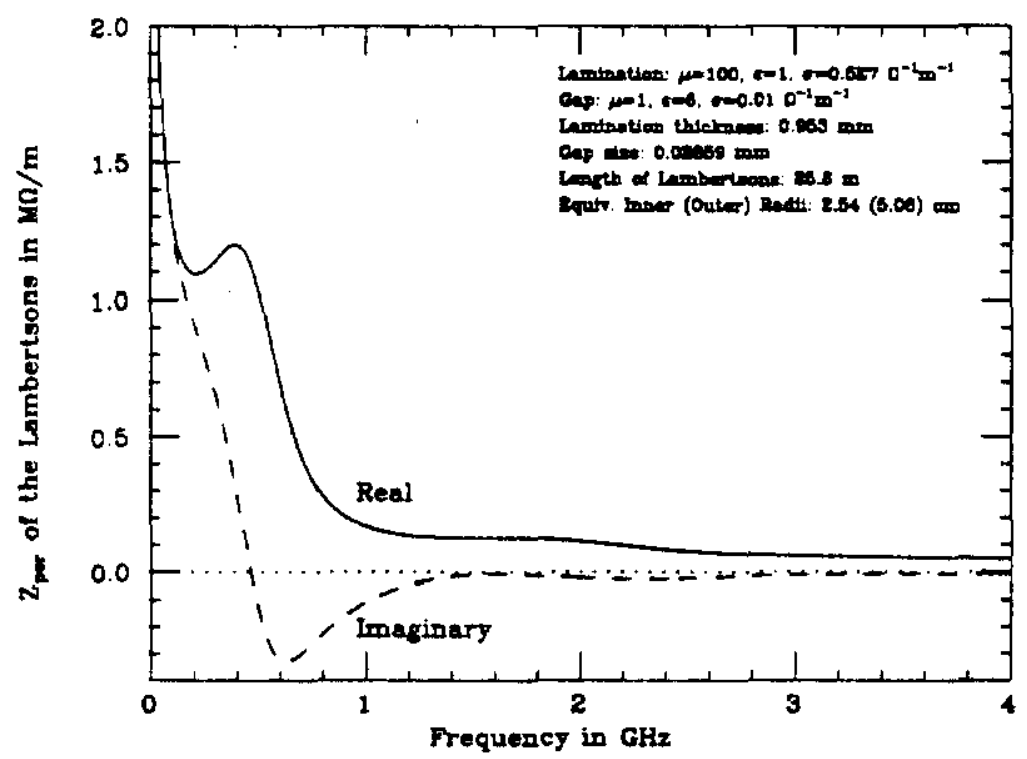

Figure 9: Real and imaginary part of the transverse impedance for the Lambertson magnets. 
peak current in the bunch $I_{p}$

$$
\left|\frac{Z_{11}}{n}\right| \leq \frac{2 \pi|\eta|(E / e) \beta^{2}}{I_{p}}\left(\frac{\sigma_{p}}{p}\right)^{2} .
$$

Here $\eta=\gamma_{t}^{-2}-\gamma^{-2}$ is the frequency slip parameter where $\gamma_{t}=20.4$ is the transition gamma. The peak current for a short bunch with a Gaussian distribution in longitudinal phase space is related to the rms bunch length $\sigma_{\tau}$ via

$$
I_{p}=\frac{N_{b} e}{\sqrt{2 \pi} \sigma_{\tau}}
$$

where $N_{b}$ is the number of particles per bunch.

Also using a short bunch approximation and a Gaussian distribution the rms momentum spread and bunch length sigma are related to the $95 \%$ normalized longitudinal emittance $S$ via

$$
S=\frac{6 \pi \beta^{3}}{\omega_{0}} \sqrt{\frac{2 \pi|\eta| E^{3}}{h e V}}\left(\frac{\sigma_{p}}{p}\right)^{2}=6 \pi \beta \omega_{o} \sqrt{\frac{h e V E}{2 \pi|\eta|}}\left(\sigma_{\tau}\right)^{2} .
$$

Substituting Equations 34 and 35 into Equation 33 we arrive at

$$
\left|\frac{Z_{\|}}{n}\right| \leq \frac{2 \pi}{\beta^{5} e I_{b}}\left(\frac{\omega_{o} S}{6 \pi \beta^{2}}\right)^{3 / 2}\left(\frac{h e V|\eta|^{3}}{8 \pi^{3} E^{3}}\right)^{1 / 4}
$$

where $I_{b}=N_{b} e \omega_{o} / 2 \pi$ and $\omega_{o} / 2 \pi$ is the revolution frequency. From this equation we can see that, except near transition where $|\eta|$ is close to zero, the impedance limit is most restrictive at high energy and low voltage.

The impedance budget for the Main Injector is calculated at injection energy of $8.9 \mathrm{GeV}$ and flattop energy of $150 \mathrm{GeV}$ with $6 \times 10^{10}$ protons per bunch. At injection we use a $95 \%$ normalized longitudinal emittance of $0.1 \mathrm{eV}$-s while at flattop we use an emittance of $0.25 \mathrm{eV}$-s. In both cases, the 


\begin{tabular}{|c|c|c|}
\hline & $8.9 \mathrm{GeV}$ & $150 \mathrm{GeV}$ \\
\hline$\left|Z_{\|} / n\right|$ & $36.9 \Omega$ & $6.6 \Omega$ \\
\hline$R_{\|} / Q$ & $85.4 \mathrm{k} \Omega$ & $26.7 \mathrm{k} \Omega$ \\
\hline$\left|Z_{\perp} / n\right|$ & $0.055 \mathrm{M} \Omega / \mathrm{m}$ & $0.63 \mathrm{M} \Omega / \mathrm{m}$ \\
\hline$\left|Z_{\perp}\right|$ & $500 \mathrm{M} \Omega / \mathrm{m}$ & $10.1 \mathrm{G} \Omega / \mathrm{m}$ \\
@ Freq. & $0.81 \mathrm{GHz}$ & $1.44 \mathrm{GHz}$ \\
\hline$R_{\perp} / Q$ & $71.6 \mathrm{M} \Omega / \mathrm{m}$ & $1450 \mathrm{M} \Omega / \mathrm{m}$ \\
\hline
\end{tabular}

Table 9: Microwave instability impedance budget for broadband and narrowband impedances. ${ }^{\dagger}$ Transverse impedance budget $\left|Z_{\perp} / n\right|$ applies only above microwave frequencies where the wavelength is smaller than the rms bunch length. Thus $\left|Z_{\perp}\right|$ is the relevant impedance budget.

$\mathrm{RF}$ voltage $V=400 \mathrm{k} V$ has been used. The results of these calculations are shown in Table 9. The bunch lengths $\sigma_{\tau}=1.24(0.69) \mathrm{ns}$ at $8.9(150) \mathrm{Gev}$ have been used.

The growth rate in the absence of Landau damping [19] is

$$
\frac{1}{\tau_{\|}}=\left(\frac{e I_{p}\left|\eta Z_{\|}\right| n}{2 \pi \beta^{2} E}\right)^{\frac{1}{2}} \omega_{0}
$$

Near transition crossing, there is negligible spread in revolution frequency for damping. Therefore the growth is mostly driven by the space-charge impedance and the rate is directly proportional to $n$ up to frequency $\omega \approx \gamma c / b$ where $b$ is the beampipe radius.

For the case of sharp resonances, narrower that the bunch spectrum, the relevant quantity [20] is not $\left|Z_{\|} / n\right|$ but $R_{\|} / Q$ where $R_{\|}$is the shunt 
impedance of a high- $Q$ resonator. The impedance budget in this narrowband case is

$$
\frac{R_{\|}}{Q} \leq \frac{4|\eta| \beta^{2}(E / e)}{I_{b}}\left(\frac{\sigma_{\nu}}{p}\right)^{2}
$$

or, using Equation 35 and 34, the equivalent expression written in terms of the longitudinal emittance is

$$
\frac{R_{\|}}{Q} \leq \frac{2 \omega_{0}}{3 \pi \epsilon I_{b}} \sqrt{\frac{h e V|\eta|}{2 \pi E}} S .
$$

With the same beam parameters as in the broadband case the limits are calculated and shown in Table 9.

In the transverse case the microwave instability limit for broadband resonances $[20,21]$, written in terms of $I_{p}$ and $\sigma_{p} / p$, is

$$
\left|Z_{\perp}\right| \leq 4 \sqrt{2 \pi} \frac{E \nu_{\beta} \beta \gamma}{e R I_{p}}\left(\frac{\sigma_{p}}{p}\right)\left|\left(n-\nu_{\beta}\right) \eta-\xi\right|,
$$

or written in terms of the longitudinal emittance $S$ is

$$
\left|Z_{\perp}\right| \leq \frac{4 \nu_{\beta} \gamma}{e R I_{b} \beta}\left(\frac{\omega_{0} S}{6 \pi}\right)\left|\left(n-\nu_{\beta}\right) \eta-\xi\right|
$$

where $\xi$ is the chromaticity. The contribution to Landau damping from the revolution frequency spread and from the tune spread are included with the $\eta$ and $\xi$ terms respectively.

With the harmonic number $n$ as a factor on the right side of Equation 40 the transverse impedance budget becomes more stringent at lower frequencies. However this equation applies only to microwave frequencies which have a wavelength $\lambda$ smaller than the rms bunch length. In other words Equation 40 applies only for harmonic number $n>2 \pi /\left(\omega_{0} \sigma_{\tau}\right)$. Except near 
transition crossing where $\eta$ is small, we have mostly $\left|\left(n-\nu_{\beta}\right) \eta\right|>|\xi|$, and the contribution of chromaticity can be neglected. Therefore we calculate the transverse impedance budget for the Main Injector using Equation 40 with $n=2 \pi / \omega_{o} \sigma_{\tau}$ giving the result

$$
\left|Z_{\perp}\right| \leq 4 \sqrt{2 \pi} \frac{E \nu_{\beta} \beta \gamma|\eta|}{e R I_{p}}\left(\frac{\sigma_{p}}{p}\right) \frac{2 \pi}{\omega_{o} \sigma_{\tau}}
$$

where the rms bunch lengths $\sigma_{\tau}=1.24 \mathrm{~ns}$ and $0.69 \mathrm{~ns}$ at $8.9 \mathrm{GeV}$ and $150 \mathrm{GeV}$, respectively, have been used. Equation 42, written in terms of the longitudiral emittance becomes

$$
\left|Z_{\perp}\right| \leq \frac{8 \pi \nu_{\beta \gamma} \gamma}{e R I_{b}}\left(\frac{S \omega_{a}}{6 \pi \beta^{2}}\right)^{\frac{1}{2}}\left(\frac{h e V E|\eta|^{3}}{2 \pi}\right)^{\frac{1}{4}} .
$$

Unlike the longitudinal impedance budget which goes to zero at transition, the transverse impedance budget is still finite due to the contribution of chromaticity. As a result transverse microwave growth usually does not occur at transition.

The transverse microwave growth rate in the absence of Landau damping $[21]$ is

$$
\frac{1}{\tau_{\perp}}=\frac{e I_{p} c Z_{\perp}}{4 \pi \nu_{\beta} E}
$$

for a broadband impedance. Note that unlike the longitudinal situation this growth rate does not depend on the frequency slip parameter $\eta$.

For narrowband transverse impedances the impedance budget is given by $[20]$

$$
\frac{R_{\perp}}{Q} \leq \frac{8 \sqrt{2} \beta \gamma E \nu_{\beta}|\eta|}{e \pi R I_{b}}\left(\frac{\sigma_{p}}{p}\right),
$$


or

$$
\frac{R_{\perp}}{Q} \leq \frac{8 \sqrt{2} \gamma \nu_{\beta}}{e \pi R I_{b}}\left(\frac{S \omega_{o}}{6 \pi}\right)^{\frac{1}{2}}\left(\frac{h e V E|\eta|^{3}}{2 \pi}\right)^{\frac{1}{4}}
$$

Using the same parameters as in the longitudinal case the transverse microwave impedance budgets were calculated and are shown in Table 9. 


\section{Conclusions and Further Work}

We have made impedance estimations of the various vacuum chamber components in the Main Injector and used these estimates to analyze beam stabjlity. Impedance estimations were made for the RF cavities, beampipe bellows, vacuum valves, beam position monitors, and Lambertson magnets. Growth rates of the coupled bunch instabilities have been calculated and a microwave instability impedance budget established. The calculations made in this paper are based on the Main Injector design parameters listed in Table 10 and we have summarized the results in Tables 11-14.

Although we find nothing to prevent the Main Injector from reaching its design goals, there are several areas of concern which should be investigated further. In particular, the issue of possible beam loss and/or emittance growth during transition crossing was not examined and requires further analysis. The possibility of a short batch coupled bunch instability during Main Injector coalescing cycles also exists. Since this type of instability is observed in the Main Ring, work should begin on designing and testing an active feedback damper to cure any potential problems.

The space charge impedance for the Main Injector was calculated using both a circular beampipe with $2.39 \mathrm{~cm}$ radius and $4.78 \mathrm{~cm}$ by $12.0 \mathrm{~cm}$ rectangular beampipe [1]. The beam size, determined from the transverse emittance and the average beta, was chosen to be $2.65 \mathrm{~mm}$. The space charge impedance is most important in estimating emittance blowup at transition. Although we have not considered transition crossing in this paper we include the space charge impedance for completeness. 


\begin{tabular}{lr}
\multicolumn{2}{c}{ Main Injector } \\
Number of particles per bunch & $6 \times 10^{10}$ \\
Number of bunches & 498 \\
Number of particles total & $3 \times 10^{13}$ \\
Longitudinal emittance at $8.9 \mathrm{GeV}$ & $0.1 \mathrm{eV}$-s \\
Longitudinal emittance at $150 \mathrm{GeV}$ & $0.25 \mathrm{eV}$-s \\
Transverse emittance in $x$ and $y$ & $20 \pi$ mm-mrad \\
RF voltage at 8.9 Gev & $400 \mathrm{kV}$ \\
RF voltage at $150 \mathrm{Gev}$ & $400 \mathrm{kV}$
\end{tabular}

\section{Main Ring}

Number of particles per buncl

$3 \times 10^{10}$

Number of bunches

1008

Number of particles total

$3 \times 10^{13}$

Longitudinal emittance at $8.9 \mathrm{GeV}$

$0.1 \mathrm{eV}-\mathrm{s}$

Longitudinal emittance at $150 \mathrm{GeV}$

$0.25 \mathrm{eV}-\mathrm{s}$

R.F voltage at $8.9 \mathrm{Gev}$

$400 \mathrm{kV}$

RF voltage at $150 \mathrm{Gev}$

$400 \mathrm{kV}$

Table 10: Parameters used for instability threshold calculations. Emittances are $95 \%$ normalized values. 


\begin{tabular}{|c|c|c|}
\hline $\begin{array}{c}\text { Space Charge } \\
\text { Impedance } \\
\text { at } 8.9 \mathrm{GeV}\end{array}$ & $\begin{array}{c}\text { Longitudinal } \\
\text { Transverse Horz } \\
\text { Transverse Vert }\end{array}$ & $\begin{array}{c}Z_{\|} / n=-j 12.3 \Omega \\
Z_{\perp x}=-j 314 \mathrm{M} \Omega / \mathrm{m}\end{array}$ \\
$Z_{\perp y}=-j 302 \mathrm{M} \Omega / \mathrm{m}$
\end{tabular}

Table 11: Summary impedance estimations and instability growth times for the Main Injector. 


\begin{tabular}{|c|c|c|c|c|c|}
\hline \multirow{2}{*}{ Freq $(\mathrm{MHz})$} & \multirow{2}{*}{$Z_{\|}(\mathrm{k} \Omega)$} & \multicolumn{3}{|c|}{ growth time in msec } \\
\cline { 3 - 6 } & \multicolumn{2}{|c|}{ dipole } & \multicolumn{2}{|c|}{ quadrupole } \\
\cline { 3 - 6 } & 10.0 & 20.2 & 50.9 & 293 & 483 \\
100.0 & 2.5 & 65.5 & 177 & 461 & 797 \\
128.0 & 6.3 & 24.2 & 71.6 & 98.7 & 182 \\
223.0 & 111.0 & 2.1 & 10.3 & 2.1 & 5.5 \\
600.0 & 277.0 & 9.1 & 32.7 & 3.5 & 20.8 \\
850.0 & 49.0 & 91.5 & 350 & 45 & 241 \\
\hline
\end{tabular}

Table 12: Dipole and quadrupole mode growth times for both the Main Injector and the Main Ring due to the longitudinal RF cavity modes.

The space charge forces are also responsible for the coherent and incoherent tune shifts [4]. These are largest at $8.9 \mathrm{GeV}$ so calculations were made at this energy. Using a 4.78 by $12.0 \mathrm{~cm}$ elliptical beampipe and a conformal mapping technique [6] the tune shifts were calculated in both planes and are shown in Table 3. The coherent tune shifts were found to be less than -0.025 and are not expected to create any problems. The incoherent tune shifts on the other hand are calculated to be rather large, especially the vertical incoherent tune shift of $\Delta_{\text {inc }}^{\mathrm{V}}=-0.086$. Note that if the Main Injector is operated with a nominal tune of 0.4 then due to the incoherent tune shift some of the particles will have a tune of $0.4-0.086=0.314$ which crosses the third integer resonance. It is not understood what, if any, problems this 


\begin{tabular}{|c|c|c|c|}
\hline $\begin{array}{c}\text { Bellows } \\
\text { (516 bellows) }\end{array}$ & $\begin{array}{r}4 \mathrm{GHz} \\
8.5 \mathrm{GHz} \\
8 \mathrm{GHz}\end{array}$ & $\begin{aligned}\left|Z_{\|} / n\right| & =4.7 \Omega \\
\left|Z_{\|} / n\right| & =0.66 \Omega \\
\left|Z_{\perp} / n\right| & =8.7 \mathrm{k} \Omega / \mathrm{m}\end{aligned}$ & $\begin{aligned} R_{\|} / Q & =38.9 \mathrm{k} \Omega \\
R_{\|} / Q & =14.4 \mathrm{k} \Omega \\
R_{\perp} / Q & =146 \mathrm{k} \Omega / \mathrm{m}\end{aligned}$ \\
\hline $\begin{array}{l}\text { Vacuum valves } \\
\text { with pillbox } \\
\text { (30 valves) }\end{array}$ & $\begin{array}{l}1.51 \mathrm{GHz} \\
2.46 \mathrm{GHz} \\
1.52 \mathrm{GHz} \\
3.11 \mathrm{GHz} \\
1.93 \mathrm{GHz} \\
3.15 \mathrm{GHz}\end{array}$ & $\begin{aligned}\left|Z_{\|} / n\right| & =135 \Omega \\
\left|Z_{\|} / n\right| & =129 \Omega \\
\left|Z_{\perp x} / n\right| & =3.0 \mathrm{k} \Omega / \mathrm{m} \\
\left|Z_{\perp_{x}} / n\right| & =6 \Omega / \mathrm{m} \\
\left|Z_{\perp y} / n\right| & =650 \Omega / \mathrm{m} \\
\left|Z_{\perp y} / n\right| & =178 \Omega / \mathrm{m}\end{aligned}$ & $\begin{aligned} R_{\|} / Q & =1090 \Omega \\
R_{\|} / Q & =924 \Omega \\
R_{\perp x} / Q & =17 \mathrm{k} \Omega / \mathrm{m} \\
R_{\perp x} / Q & =0.53 \mathrm{k} \Omega / \mathrm{m} \\
R_{\perp y} / Q & =7.2 \mathrm{k} \Omega / \mathrm{m} \\
R_{\perp y} / Q & =1.6 \mathrm{k} \Omega / \mathrm{m}\end{aligned}$ \\
\hline $\begin{array}{l}\text { Vacuum valves } \\
\text { without } \\
\text { pillbox } \\
\text { (30 valves) }\end{array}$ & $\begin{array}{l}1.30 \mathrm{GHz} \\
2.46 \mathrm{GHz} \\
1.52 \mathrm{GHz} \\
3.11 \mathrm{GHz}\end{array}$ & $\begin{aligned}\left|Z_{\|} / n\right| & =108 \Omega \\
\left|Z_{\|} / n\right| & =13 \Omega \\
\left|Z_{\perp x} / n\right| & =387 \Omega / \mathrm{m} \\
\left|Z_{\perp y} / n\right| & =28.0 \Omega / \mathrm{m}\end{aligned}$ & $\begin{aligned} R_{\|} / Q & =975 \Omega \\
R_{\|} / Q & =150 \Omega \\
R_{\perp x} / Q & =5.0 \mathrm{k} \Omega / \mathrm{m} \\
R_{\perp_{y}} / Q & =384 \Omega / \mathrm{m}\end{aligned}$ \\
\hline
\end{tabular}

Table 13: Summary of beampipe bellows and vacuum valve impedance estimations for the Main Injector. 


\begin{tabular}{|c|c|c|}
\hline $\begin{array}{l}\text { Microwave } \\
\text { Longitudinal }\end{array}$ & $8.9 \mathrm{GeV}$ & $\begin{array}{l}\left|Z_{\|} / n\right|<36.9 \Omega \\
R_{\|} / Q<84.5 \mathrm{k} \Omega\end{array}$ \\
\hline $\begin{array}{c}\text { Impedance } \\
\text { Budget }\end{array}$ & $150 \mathrm{Gev}$ & $\begin{array}{c}\left|Z_{\|} / n\right|<6.6 \Omega \\
R_{\|} / Q<26.7 \mathrm{k} \Omega\end{array}$ \\
\hline $\begin{array}{l}\text { Transverse } \\
\text { Microwave }\end{array}$ & $8.9 \mathrm{GeV}$ & $\begin{array}{c}\left|Z_{\perp} / n\right|<0.055 \mathrm{M} \Omega / \mathrm{m} \\
\left|Z_{\perp}\right|<500 \mathrm{M} \Omega / \mathrm{m} \\
@ \text { freq. } 0.81 \mathrm{GHz}^{\dagger} \\
R_{\perp} / Q<71.6 \mathrm{M} \Omega / \mathrm{m}\end{array}$ \\
\hline $\begin{array}{l}\text { Impedance } \\
\text { Budget }\end{array}$ & $150 \mathrm{GeV}$ & $\begin{array}{c}\left|Z_{\perp} / n\right|<0.63 \mathrm{M} \Omega / \mathrm{m} \\
\left|Z_{\perp}\right|<10.1 \mathrm{G} \Omega / \mathrm{m} \\
@ \text { freq. } 1.44 \mathrm{GHz}^{\dagger} \\
R_{\perp} / Q<1450 \mathrm{M} \Omega / \mathrm{m}\end{array}$ \\
\hline
\end{tabular}

Table 14: Summary of the microwave instability impedance budget in the Main Injector. ' For the transverse broadband impedance the threshold $\left|Z_{\perp} / n\right|$ is calculated but only applies when the wavelength is shorter than the bunch length. Therefore the relevant value is $\left|Z_{\perp}\right|$ at the frequency where the wavelength equals the bunch length. 
will cause.

Using the measured longitudinal impedance values $[7,8]$, the coupled bunch growth times for the different higher order RF modes were calculated using the standard perturbation theory [11]. Although the calculated growth times are short we do not expect coupled bunch instabilities to limit Main Injector performance. First, the above estimates do not include Landau damping effects which help stabilize the beam. Secondly, the calculation assumes the frequencies of the higher order modes for all $18 \mathrm{RF}$ cavities are exactly the same. The differences between cavities will reduce the net impedance. Finally, similar calculations in the Main Ring also show short growth times but the instability is not the limiting factor in the Main Ring. To alleviate any potential problems a set of passive mode dampers were installed in the RF cavities to reduce shunt impedances of several higher order modes. If it becomes necessary the shunt impedance of the other modes can be reduced as well.

For short batches which do not completely fill the ring, as in coalescing and antiproton production cycles, the standard techniques for calculating growth times do not apply. Instead we rely on operational experience in the Main Ring. Presently there are signs of a short batch instability in the Main Ring during coalescing cycles in which 11 consecutive bunches of $1.5 \times 10^{10}$ protons per bunch are accelerated. The Main Injector, having even higher beam intensities of $6 \times 10^{10}$ protons per bunch, will have the same problem. Since this type of instability depends on the $R / Q$ value of the higher order cavity mode, and not on the shunt impedance $R$, passive mode dampers, which reduce $R$ but leave $R / Q$ unchanged, cannot be used 
to cure this instability. The solution is the design and construction of an active narrowband damping system. Since the problem exists in the Main Ring work is in progress on designing such a system.

Another potential area of concern is the transverse coupled bunch instability due to the resistive wall and Lambertson impedances. Using estimated impedances the calculated growth time $[11,12]$ for this instability, not including Landau damping effects, was found to be $0.35 \mathrm{~ms}$ in the Main Injector and, as a comparison, $0.65 \mathrm{~ms}$ in the Main Ring. Even though the growth time in the Main Ring is short, the transverse dampers in the Main Ring are presently capable of damping out this instability. Therefore it is thought that the present system, possibly modified to increase the gain, should be capable of preventing the resistive wall instability in the Main Injector.

Observations in the Main Ring [14] also suggest that using sextupoles and octupoles is effective in eliminating the resistive wall instability even with the dampers turned off. Therefore we conclude that resistive wall instabilities should not limit Main Injector intensity.

The impedance of the beam position monitors was calculated by treating the pickups as striplines and estimating the fraction of image current that enters these striplines. It is then possible to relate the stripline impedance to the beam impedance. Even with a total of 208 BPMs in the Main Injector the net impedance is small and will not affect operations.

The impedance of the vacuum valves was calculated using the program MAFIA [18] and two different designs were considered. One design has two small "pillbox" cavities on either side of the vacuum valve while the other does not. It was found that the non-pillbox design has lower impedances than 
the pillbox design although with 30 valves both are within the impedance budget limits.

Estimates of the bellows impedance was made using TBCI [17] with a circular beampipe and circular bellows. The calculations show a few modes at higher frequencies but the net impedance of the 508 bellows is not expected to cause problems with the possible exception of at transition.

The low frequency impedance of the Lambertson magnets was estimated by calculating the net resistance seen by the image current traveling through the magnet laminations. The laminations were approximated as annular rings with an inner radius of $2.54 \mathrm{~cm}$. It should be noted that this is only a rough approximation. The actual shape of the Lambertson aperture is not circular and the transverse impedance varies roughly as the inverse of the radius cubed. Therefore a $10 \%$ increase in the radius will reduce the estimations of the longitudinal impedance by $10 \%$ and the transverse impedance by $25 \%$. In the transverse plane the Lambertson impedance contributes about one half of the total resistive wall impedance.

Finally we calculate the impedance budget for the microwave instability at $8.9 \mathrm{GeV}$ and $150 \mathrm{GeV}$. The impedance budget for the microwave instability differs for broadband and narrowband impedances $[19,20]$. The usual broadband budgets are expressed as $\left|Z_{\| \mid} / n\right|$ and $\left|Z_{\perp}\right|$ whereas the narrowband budget is determined by the value of $R / Q$ where $R$ is the shunt impedance of the narrowband impedance and $Q$ is the quality factor. Impedances such as space charge should be less than the broadband budget and impedances such as RF cavities and vacuum valves should be less than the narrowband limit. 
The bellows longitudinal impedances meet both the broadband microwave impedance budget and the narrowband budget. Note that the longitudinal and transverse impedances of the bellows have been treated as broadband because the resonances have a $Q$ of $\approx 5$ and are above cutoff.

Although an important topic, transition crossing limitations have not been addressed in this paper. Space charge, negative mass and microwave instabilities at transition can potentially limit beam intensity or blow up beam emittance. Further work should look into transition crossing.

Also important is the short batch coupled bunch instability caused by the higher order RF cavity modes and presently observed in the Main Ring. The design and implementation of an active damping feedback loop should be pursued.

We have not estimated the impedance of the kicker magnets. We also have not studied the possibility of slow head-tail instability and bunch modecoupling instabilities. These issues may be important and will be investigated elsewhere. 


\section{References}

[1] K. Ng, "Exact Solutions for the Longitudinal and Transverse Impedances of an Off-centered Beam in a Rectangular Beam Pipe," Particle Accelerators, vol. 16, pp. 63-80, 1984.

[2] L. Laslett, "On Intensity Limitations Imposed by Transverse SpaceCharge Effects in Circular Particle Accelerators," in 1963 Summer Study on Storage Ring Accelerators and Experimentation at Super High Energies, BNL-753 , pp. 324-367, 1963.

[3] L. Laslett, " The Space-Charge Intensity Limit Imposed by Coherent Oscillation of a Bunched Synchrotron Beam," in Proceedings 6th International Conference of High Energy Accelerators, CEAL-2000, p. 150, 1967.

[4] P. Bryant, "Betatron Frequency Shifts Due to Self and Image Fields," in CERN Accelerator School, CERN 87-10, 1987.

[5] D.A. Edwards and M.J. Syphers, An Introduction to the Physics of High Energy Accelerators. New York: John Wiley and Sons, Inc., 1993.

[6] B. Zotter, "The Q-Shift of Off-Center Particle Beams in Elliptic Vacuum Chambers," Nucl. Instrum. Methods, vol. 129, pp. 377-395, 1975.

[7] R.A. Dehn, Q.A. Kerns, and J.E. Griffin, "Mode Damping in NAL Main Ring Accelerating Cavities," IEEE Trans. Nucl. Sci., vol. NS-18, pp. 260-261, 1971. 
[8] Q. Kerns and H. Miller, "Fermilab 500-Gev Main Accelerator RF Cavity 128-MHz Mode Damper," IEEE Trans. Nucl. Sci, vol. NS-24, pp. 17041706,1971 .

[9] P. Colestock and S. Saritepe, "Transverse Instability Estimates in the Fermilab Booster and Main Ring," in Proceedings of the Fermilab III Instabilities Workshop, pp. 235-244, 1990.

[10] U. Lauströer, U. van Rienen, and T. Weiland, "URMEL and URMEL-T User Guide," Tech. Rep. M-87-03, DESY, 1987.

[11] J. Laclare, "Bunched Beam Coherent Instabilities," in CERN Accelerator School, CERN 87-03, 1985.

[12] F. Sacherer, "A Longitudinal Stability Criterion for Bunched Beams," IEEE Trans. Nucl. Sci., vol. NS-20, pp. 825-829, 1973.

[13] B. Zotter and F. Sacherer, "Transverse Instabilities of Relativistic Particle Beams in Accelerators and Storage Ring," CERN 77-13, pp. 175-218, 1977 .

[14] S. Pruss. Private communication. Fermilab.

[15] Los Alamos Accelerator Code Group, "Reference Manual for the POISSON/SUPERFISH Group of Codes," tech. rep., Los Alamos, 1987.

[16] W.K.H. Panofsky and W.A. Wenzel, "Some Considerations Concerning the Transverse Deflections of Charged Particles in Radio-Frequency Fields," Rev. Sci. Instrum., vol. 26, p. 967, 1956. 
[17] R. Klatt and T. Weiland, TBCI Short User Guide. DESY, 1988.

[18] DESY, LANL and KFA, MAFIA User Guide, 1989.

[19] S. Krinsky and J.M. Wang, "Longitudinal Instabilities of Bunched Beams Subject to a Non-Harmonic RF Potential," Particle Accelerators, vol. 17, p. 109, 1985.

[20] K. Ng, "Microwave Instability When Driven By Narrow Resonances," in Proceedings of the 1986 Summer Study on the Physics of the Superconducting Super Collider, (Snowmass, Colorado), June 1986.

[21] R.D. Ruth and J.M. Wang, "Vertical Fast Blow-up in a Single Bunch," IEEE Trans. Nucl. Sci., vol. NS-28, pp. 2405-2407, 1981. 